\title{
Remanence anisotropy effect on the palaeointensity results obtained from various archaeological materials, excluding pottery
}

\author{
Mary Kovacheva ${ }^{1}$, Annick Chauvin $^{2}$, Neli Jordanova ${ }^{1}$, Philippe Lanos ${ }^{2,3}$, and Vassil Karloukovski ${ }^{1,4}$ \\ ${ }^{1}$ Geophysical Institute, Bulgarian Academy of Sciences, Sofia, Bulgaria \\ ${ }^{2}$ Geosciences-Rennes, UMR 6118 CNRS-University of Rennes 1, France \\ ${ }^{3}$ IRAMAT, CNRS, UMR 5060, Université de Bordeaux 3, France \\ ${ }^{4}$ Centre for Environmental Magnetism, Geographical Department of Lancaster University, Lancaster LAI 4YB, U.K.
}

(Received December 12, 2007; Revised October 26, 2008; Accepted November 15, 2008; Online published July 27, 2009)

\begin{abstract}
The effect of magnetic anisotropy on the palaeointensity results has been evaluated in different materials, including samples from archaeological structures of various ages, such as baked clay from prehistoric domestic ovens or pottery kilns, burnt soil from ancient fires, and bricks and bricks or tiles used in the kiln's construction. The remanence anisotropy was estimated by the thermoremanent (TRM) anisotropy tensor and isothermal remanence (IRM) tensor methods. The small anisotropy effect (less than 5\%) observed in the palaeointensity results of baked clay from the relatively thin prehistoric oven's floors estimated previously through IRM anisotropy was confirmed by TRM anisotropy of this material. The new results demonstrate the possibility of using IRM anisotropy evaluation to correct baked clay palaeointensity data instead of the more difficult to determine TRM anisotropy ellipsoid. This is not always the case for the palaeointensity results from bricks and tiles. The anisotropy correction to palaeointensity results seems negligible for materials other than pottery. It would therefore appear that the palaeointensity determination is more sensitive to the degree of remanence anisotropy $P$ and the angle between the natural remanent magnetization (NRM) vector and the laboratory field direction, than to the angle between the NRM and the maximum axis of the remanence anisotropy ellipsoid $\left(K_{\max }\right)$.
\end{abstract}

Key words: Archaeomagnetism, baked clay materials, palaeointensity, magnetic anisotropy correction.

\section{Introduction}

Palaeointensity determination remains one of the most difficult tasks in palaeo- and archaeomagnetism. Palaeointensity techniques are based on a comparison between natural remanent magnetization (NRM) and an artificial magnetization given in a known laboratory field. The influence of magnetic anisotropy on the palaeointensity evaluation was suggested long time ago, and the notion of 'easy plane' was introduced, especially in the case of pottery (Rogers et al., 1979). To avoid the anisotropy effect Aitken et al. (1981) adjusted the direction of the natural remanent magnetization (NRM) of their samples as close as possible to the direction of the applied laboratory field. Veitch et al. (1984) suggested the determination of the thermoremanent magnetization anisotropy ellipsoids for samples of tiles and bricks. In the studies reported by Yang et al. (1993a, b) and Selkin et al. (2000), the thermoremanent anisotropy tensor was replaced by that of an induced anhysteretic remanence (ARM) tensor. Hus (2001) and Hus et al. (2002) showed that the partial anhysteretic remanence (pARM) anisotropy ellipsoid mimics best the TRM anisotropy ellipsoid and can be used for the correction of palaeointensity determinations. Such corrections have been applied by Yang et al. (1993b), Selkin et al. (2000) and Carvallo and Dunlop

Copyright (c) The Society of Geomagnetism and Earth, Planetary and Space Sciences (SGEPSS); The Seismological Society of Japan; The Volcanological Society of Japan; The Geodetic Society of Japan; The Japanese Society for Planetary Sciences; TERRAPUB
(2001). The general disadvantage to this approach is that the ARM has to be induced after the palaeointensity experiment and that the magnetic fraction carrying the ARM can be different from the one carrying the TRM due to mineralogical changes that occur during heating (Henry et al., 2003).

In contrast to the substantial effect of remanence anisotropy on the palaeointensity estimates from rocks with a significant petrofabric described in Selkin et al. (2000), Yu et al. (2001) reported a very weak influence of the remanence anisotropy on the palaeointensity estimates from a Tudor Gabbro, Ontario.

Garcia (1996) and Chauvin et al. (2000) studied the effect of anisotropy on the palaeointensity results by estimating the thermoremanent magnetization (TRM) tensor using samples from ancient bricks used in the kiln's construction. Their studies revealed that the anisotropy effect is quite important and not at all negligible reaching, in some cases, a correction factor of $25 \%$ of the determined field strength. The anisotropy effect on the palaeointensity result estimated through the anisotropy of induced isothermal remanent magnetisation (IRM), as recommended in Stephenson et al. (1986), has been previously performed for samples of baked clay of prehistoric ovens. This effect was found to be negligible, and the correction factor " $f$ " of the absolute palaeointensity values is usually lower than $5 \%$ (Jordanova et al., 1995; Jordanova, 1996; Kovacheva et al., 1998). The idea of substituting the determination of the TRM anisotropy tensor by the determination of the IRM 
anisotropy tensor without heating has practical advantages, but needs confirmation.

Our aim was, therefore, to compare the anisotropy correction to palaeointensity results through IRM- and TRMinduced remanence on the same material. To this end, we used archaeological samples of various origins and ages. The palaeointensity study was performed using the classical Thellier method (Thellier and Thellier, 1959) in two laboratories (the Geosciences Laboratory, University of Rennes 1, France and the Palaeomagnetic laboratory of the Geophysical Institute, Bulgarian Academy of Sciences, Sofia). In addition to other factors involved in the palaeointensity determination, such as the non-linearity of the Arai diagram, mineralogical changes, non-suitable magnetic characteristics, among others (Chauvin et al., 1991; Jordanova et al., 1997; Kovacheva et al., 1998; Jordanova et al., 2003), a detailed examination of the magnetic anisotropy effect on the palaeointensity results obtained from materials other than pottery is still required.

Here, we present multiple measurements of the magnetic anisotropy of 85 archaeomagnetic samples of different materials. Three large tables containing all of the calculated parameters of magnetic thermoremanence anisotropy measurements at different temperature steps and of the induced isothermal remanence are given.

\section{Experimental Set-up \\ 2.1 Samples}

The collection of samples studied here comes from two areas (France and Bulgaria) and consists of 85 samples from baked clay floors of ovens and kilns and burnt soil of ancient fires (BC-BS), bricks as displaced material and bricks or tiles in kiln's construction (BR-TL). Bricks as displaced material presumably carry univectorial remanence. Bricks or tiles used in the construction of pottery kilns can carry a two-vector remanence when their heating as a constructive element of a kiln was not sufficient to entirely replace the primary magnetization, obtained in different position during their fabrication. Nevertheless, the effect of the shape anisotropy on the initial magnetization and that obtained during re-heating should be the same.

Samples coming from Bulgarian sites have a cubic shape with an edge of $20 \mathrm{~mm}$ or $24 \mathrm{~mm}$. Rarely-when the quantity of archaeomagnetic material was insufficient- the cubes were completed with plaster of Paris. Samples from French sites are all of standard cylindrical shape. Bricks and tiles (BR-TL) show considerable shape anisotropy (Lanos, 1987a, b; Goulpeau et al., 1989), which should affect the direction of the TRM acquired. Shape anisotropy is related to the form of the whole objects as is the case with pottery, while the anisotropy measured in the laboratory is governed by the distribution and shape of the magnetic grains. Here, we consider the effect of this latter anisotropy on the palaeointensity results, taking into account the nature of samples. It should be also mentioned that the preferential alignment of the magnetic grains carrying the remanence is in fact related to the initial form of the whole object (brick, tile, pottery), thus explaining the used term of 'easy plane'. The measured anisotropy of remanence susceptibility reflects both the shape and distribution of the magnetic grains due to the object's form. 'Easy plane' in archaeological objects corresponds to the petrofabric in rocks.

\subsection{Methodology}

Remanent magnetization was measured at Rennes with a Molspin spinner magnetometer and in Sofia with an astatic magnetometer. The classical Thellier method (Thellier and Thellier, 1959) for palaeointensity determination consists of a double heating of samples, with each temperature step performed in applied laboratory magnetic field. The remagnetization is made successively to higher temperatures, with several partial thermoremanence acquisition (pTRM) tests (Coe et al., 1978). The direction of the TRM acquired during natural (initial) cooling by objects with shape anisotropy or petrofabric deviates from the local direction of the geomagnetic field, and the intensity of the TRM depends on the direction in which the local field is applied. During the course of the Thellier experiments, the TRM anisotropy should be taken into account if the samples are not re-magnetized in the direction of their initial TRM (Odah et al., 2001). Because of the fact that this condition is rarely met in the usual practice, we aim to quantitatively evaluate the possible errors for materials different from potteries.

The anisotropy effect on the palaeointensity result has been studied both using the TRM anisotropy evaluation, performed twice for most of the samples, and the IRM anisotropy evaluation on the same samples, performed after the Thellier experiment (in the laboratories in both Sofia and Rennes). Both a Schonstedt static AF demagnetizer (in Rennes) and a thumbling Molspin AF demagnetizer (in Sofia) were used.

\subsection{TRM anisotropy determination}

The determination of the TRM anisotropy tensor was done first alongside the usual procedure of the Thellier experiment at temperatures corresponding to known major unblocking temperatures of NRM. To ensure that the new remanence was obtained by the un-altered assemblage of magnetic grains, these temperatures were taken between 430 and $450^{\circ} \mathrm{C}$ if positive (pTRM) tests were observed. In order to evaluate the TRM anisotropy, we performed five additional heatings at the chosen temperature step at which the samples had already been re-magnetized along their $\pm Z$ axes. The additional four re-magnetizations were along the $\pm X$ and $\pm Y$ axes. Finally, a last re-magnetization was performed along the $+Z$ axis of the samples in order to check their thermal stability. If no or few modifications of the TRM acquisition capacity were observed, the TRM anisotropy tensor was then determined (Chauvin et al., 2000), otherwise the sample was rejected. Due to the fact that the first determination of thermoremanent anisotropy (TRM1) was evaluated during several palaeointensity batches/runs of BC-BS and BR-TL samples, the temperature of this evaluation differed for different batches, and this is noted accordingly in tables and figures. At the end of the Thellier experiment (temperature of 550 or $620^{\circ} \mathrm{C}$ ), when almost all the original NRM was removed, the thermoremanent anisotropy tensor (TRM2) was evaluated once again.

Anisotropy parameters describing the TRM tensor were calculated (the principal axes denoted as $K_{\max }, K_{\text {int }}$, and 


\section{Degree of TRM anisotropy}

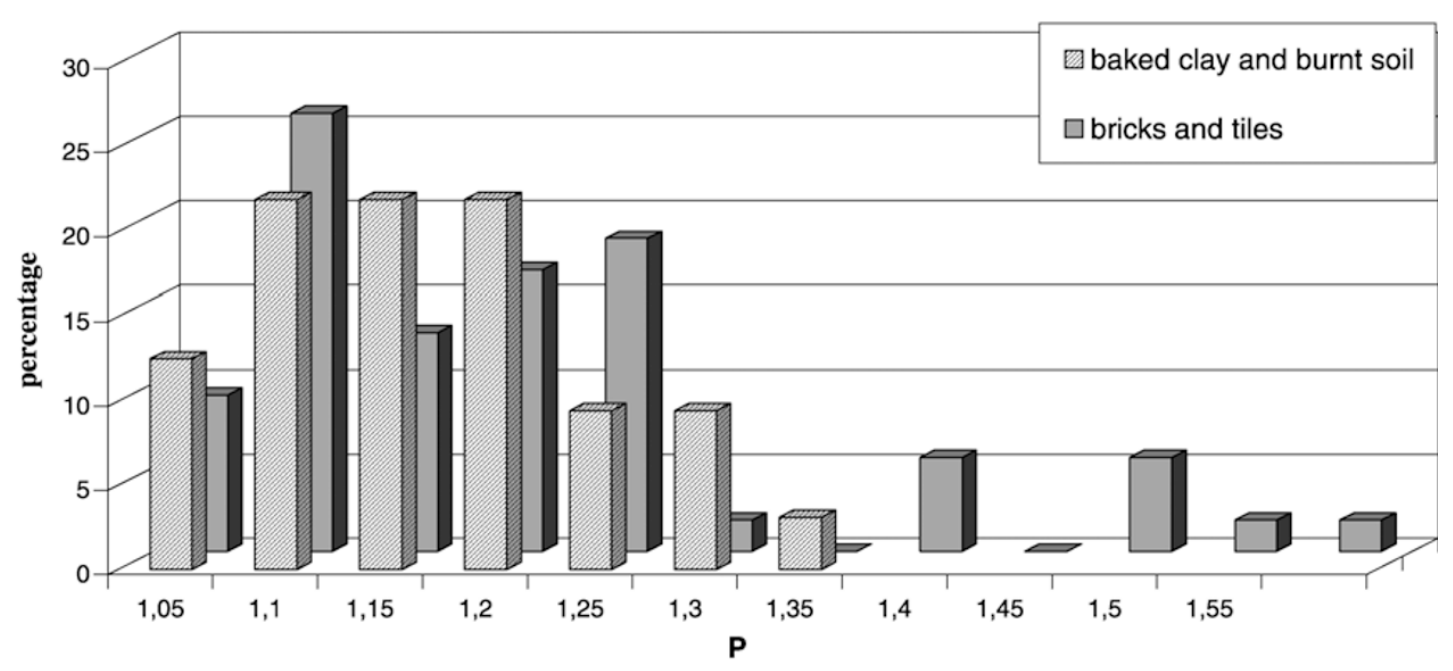

Fig. 1. Histogram showing the frequency distribution in percentage of the measured TRM1 degree of anisotropy, obtained by the samples from baked clay/burnt soils (BC-BS) and the same for bricks/tiles (BR-TL) samples.

$\left.K_{\min }\right)$, as were their orientations in sample co-ordinates. The anisotropy parameters consisted of: lineation ( $L=$ $\left.K_{\max } / K_{\text {int }}\right)$, foliation $\left(F=K_{\text {int }} / K_{\min }\right)$, the shape parameter $\left(T=\left(2 \eta_{2}-\eta_{1}-\eta_{3}\right) /\left(\eta_{1}-\eta_{3}\right)\right.$, where $\eta_{1}=\ln K_{\max }$, $\left.\eta_{2}=\ln K_{\text {int }}, \eta_{3}=\ln K_{\min }\right)$, and the degree of anisotropy $\left(P=K_{\max } / K_{\min }\right)$ (Jelinek, 1981; Hrouda, 1982; Stephenson et al., 1986). Both vectors (NRM remained and pTRM gained) were corrected at each temperature step of the Thellier experiment. Thus, the corrected Arai diagram (Nagata et al., 1963) and the corrected palaeointensity value $\left(H_{\text {acor }}\right)$ were obtained. The correction factor $f$ is the ratio $H_{\text {acor }} / H_{\mathrm{a}}$, where $H_{\mathrm{a}}$ is the palaeointensity value without correction for remanence anisotropy. $f$ can also be expressed as the percentage difference from the non-corrected palaeointensity value (thus $f=1.04$ corresponds to a $4 \%$ difference of the $H_{\text {acor }}$ from $H_{\mathrm{a}}$ ).

\subsection{IRM anisotropy determination}

The anisotropy of IRM was calculated on the same samples as those used in the Thellier experiment, after the last heating step. A steady magnetic field of $60 \mathrm{mT}$ was applied, inducing IRM successively along $\pm X, \pm Y$, and $\pm Z$ axes of the specimen. While working with archaeological materials, Marton (1996) mentioned that if a low steady field is used, the IRM ellipsoid should be dominated by multidomain (MD) particles. Thus, an intensity of $50-60 \mathrm{mT}$ is necessary (Stephenson et al., 1986; Tarling and Hrouda, 1993 , p. 81 ) to ensure that the anisotropy ellipsoid is determined on the single domain (SD) particles, which carry almost all of the NRM. At the same time, the field should not be higher in order to remain at the so-called region of Rayleigh where the IRM is proportional to the square of the applied field (Daly and Zinsser, 1973). Between the successive magnetizations, the samples are demagnetized by an alternating field (AF) of $100 \mathrm{mT}$. The residual magnetization observed after AF demagnetization appears to be negligible compared to the intensity of the IRM acquired, which is consistent with the prevailing low magnetic coer- civity of our samples (MDF being in the range of 15-25 mT for most of them).

Following the proposal of Veitch et al. (1984), we defined a unit vector $\boldsymbol{h}$ of the ancient magnetic field by:

$$
\boldsymbol{h}=\left(K_{\mathrm{I}}\right)^{-1} \cdot \mathbf{N R M} /\left|\left(K_{\mathrm{I}}\right)^{-1} \cdot \mathbf{N R M}\right|,
$$

where $\left(K_{\mathrm{I}}\right)^{-1}$ is the inverse anisotropy tensor of the laboratory-induced IRM.

Then, the correction factor $f$ is the ratio of IRM acquired by the unit field parallel to the direction of the known laboratory field during the experiment and IRM acquired by the unit field parallel to $\boldsymbol{h}$. Or:

$$
f=\left|\left(K_{\mathrm{I}}\right) \cdot \boldsymbol{l}\right| /\left|\left(K_{\mathrm{I}}\right) \cdot \boldsymbol{h}\right|
$$

where $\boldsymbol{l}$ is the unit vector along the laboratory field.

Principal axes of the corresponding IRM tensors and anisotropy parameters are denoted the same as those for the TRM anisotropy, defined above.

\section{Experimental Results}

Taking into account the different nature of the studied samples, the results are given separately for baked clay/burnt soils (BC-BS) and for bricks/tiles (BR-TL). The relationship between the anisotropy parameters obtained at different temperature steps during the palaeointensity experiment (TRM1 and TRM2) and between parameters obtained by the thermoremanent anisotropy ellipsoid and the IRM one is considered.

3.1 TRM anisotropy evaluation at two temperatures

The degree of TRM1 anisotropy $(P)$ of 33 BC-BS samples measured at an intermediate temperature (428 or $450^{\circ} \mathrm{C}$ ) is relatively weak (Fig. 1). Of the samples studied, $12 \%$ have $P \leq 1.05$ and $67 \%$ have $1.05<P \leq 1.2$. There is only one case with $P=1.31$. The degree of TRM1 anisotropy for $52 \mathrm{BR}-\mathrm{TL}$ samples (at 440 or $450^{\circ} \mathrm{C}$ ) is higher than that for the BC-BS samples (Fig. 1). We have 
a)

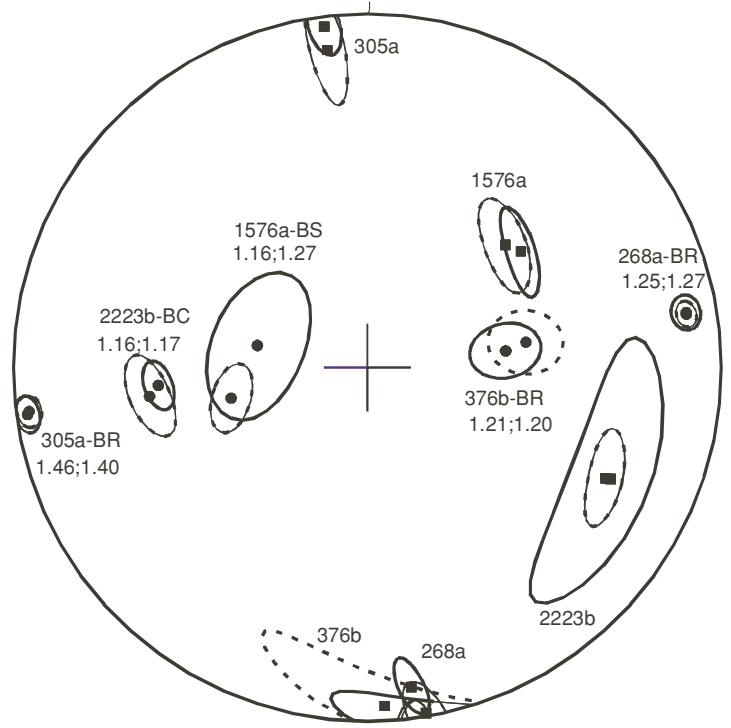

b)

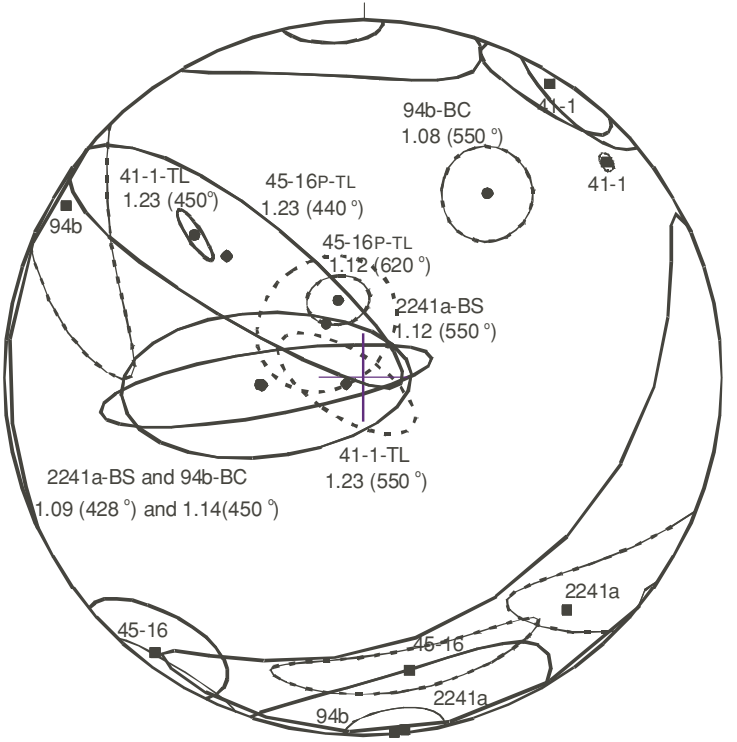

c)

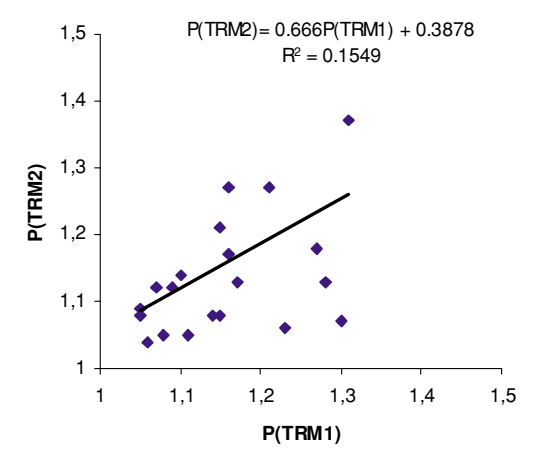

d)

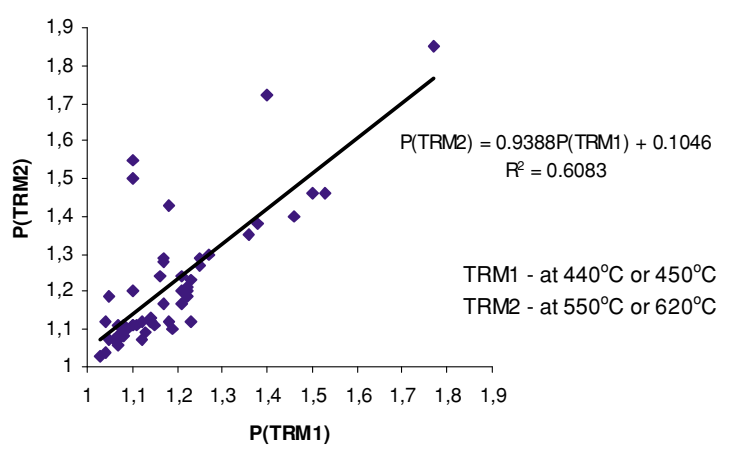

Fig. 2. Stereographic projections, in sample co-ordinates, of the principal maximal and minimal axes' directions of the TRM ellipsoids determined at two different temperatures for some typical samples; dots denote the minimal axes and squares denoted the maximal axes. (a) a good consistency; (b) bad consistency of the principal axes directions. The sample numbers and the type of material are shown together with the degree of remanence anisotropy at the two temperature levels (the second line). The stereographic projections are given in the lower hemisphere, with the confidence ellipses given with dashed lines for the higher temperature evaluation (TRM2); (c) and (d) relationships between the remanence degree of anisotropy $P$ at the two temperature levels for different type of materials.

fewer than $10 \%$ of cases with $P \leq 1.05$ and $56 \%$ in which $1.05<P \leq 1.2$, but in $32 \%$ of cases $1.2<P \leq 1.5$. In two cases, $P$ exceeds this upper limit (samples $34-15 \mathrm{P}$ and $1596 \mathrm{v}$ with a highest value of 1.77 ; Table 2 ). A total of 158 TRM anisotropy evaluations were made on the 85 samples.

One basic condition for a comparison of anisotropy tensors obtained at two different temperatures is that the same assemblages of magnetic carriers participate in the two evaluations.

In the BC-BS materials, some external factors, such as irregularities in the cube's shape, can influence the measured weaker anisotropy parameters; four prehistoric samples have been excluded because of their imperfect shape. A series of BC-BS and BR-TL samples has been subjected to a second evaluation of the anisotropy tensor at a higher temperature $\left(\mathrm{TRM} 2\right.$ at $\left.550^{\circ} \mathrm{C}\right)$, using a different sample holder, allowing for a better positioning of the samples within the oven. Additionally, 27 BR-TL samples were studied for their TRM anisotropy twice (at $440^{\circ} \mathrm{C}$ and $620^{\circ} \mathrm{C}$; Table 2 , lower part), with a very precise positioning in a specially designed holder. The coincidence of the directions of the principal remanence anisotropy axes, evaluated at two different temperature steps, is good in many cases (Fig. 2(a); samples 1576a and 2223b). For samples with stronger anisotropy degree like BR-TL, these directions are determined more precisely (Fig. 2(a); samples 305a, 376b, 268a) despite the probability of a bad positioning at the lower temperature. Samples with weaker anisotropy, however, show a very large uncertainty in terms of the directions of the principal axes (Fig. 2(b); samples 2241a, 94b). A closer look at Fig. 2(b) shows that, in most cases, the principal directions determined at the lower temperature have unacceptably large errors and cannot be considered to be real. This fact probably partly reflects the bad positioning of some samples, mentioned above using the first holder for the TRM1 anisotropy measurements at the lower tempera- 
Table 1. TRM anisotropy parameters obtained for samples of baked clay and burnt soil (BC-BS) at two temperatures: during the Thellier palaeointensity experiment $\left(428^{\circ}\right.$ or $\left.450^{\circ} \mathrm{C}\right)$ and at the end of the same experiment $\left(550^{\circ} \mathrm{C}\right)$; IRM anisotropy parameters obtained after the palaeointensity experiment by a steady field of $60 \mathrm{mT}$. The shape parameters $T$ are given below the foliation $(F)$ parameter in each case.

\begin{tabular}{|c|c|c|c|c|c|c|c|c|c|c|c|c|c|c|c|c|c|c|}
\hline \multirow{3}{*}{$\begin{array}{c}\text { No } \\
\text { material }\end{array}$} & \multicolumn{6}{|c|}{ ANISOTROPY OF TRM1 } & \multicolumn{6}{|c|}{ ANISOTROPY OF TRM2 } & \multicolumn{6}{|c|}{ ANISOTROPY OF IRM } \\
\hline & \multicolumn{3}{|c|}{428 (deg. C) } & \multirow{2}{*}{$\frac{\mathrm{L}}{\mathrm{K} 1 / \mathrm{K} 2}$} & \multirow{2}{*}{$\frac{F}{K 2 / K 3}$} & \multirow{2}{*}{$\frac{\mathrm{P}}{\mathrm{K} 1 / \mathrm{K} 3}$} & \multicolumn{3}{|c|}{550 (deg. C) } & \multirow{2}{*}{$\frac{\mathrm{L}}{\mathrm{K} 1 / \mathrm{K} 2}$} & \multirow{2}{*}{$\frac{F}{K 2 / K 3}$} & \multirow{2}{*}{$\frac{\mathrm{P}}{\mathrm{K} 1 / \mathrm{K} 3}$} & \multicolumn{3}{|c|}{$(60 \mathrm{mT})$} & \multirow{2}{*}{$\frac{\mathrm{L}}{\mathrm{K} 1 / \mathrm{K} 2}$} & \multirow{2}{*}{$\frac{F}{K 2 / K 3}$} & \multirow{2}{*}{$\frac{P}{\mathrm{~K} 1 / \mathrm{K} 3}$} \\
\hline & & D & 1 & & & & & D & 1 & & & & & D & 1 & & & \\
\hline $111 \mathrm{~b}$ & $\mathrm{~K} 1$ & 260 & 44 & 1,06 & 1,08 & 1,15 & K1 & 55 & 26 & 1,02 & 1,06 & 1,08 & K1 & 78 & 42 & 1,05 & 1,01 & 1,06 \\
\hline baked & K2 & 4 & 15 & & $\mathrm{~T}$ & & $\mathrm{~K} 2$ & 318 & 13 & & $\mathrm{~T}$ & & K2 & 252 & 47 & & $\mathrm{~T}$ & \\
\hline clay & K3 & 109 & 43 & & 0,15 & & K3 & 204 & 61 & & 0,57 & & K3 & 345 & 2 & & $-0,72$ & \\
\hline $19 \mathrm{v}$ & K1 & 238 & 46 & 1,03 & 1,06 & 1,10 & & & & & & & K1 & 223 & 63 & 1,04 & 1,01 & 1,05 \\
\hline $\begin{array}{c}\text { baked } \\
\text { clay }\end{array}$ & $\begin{array}{l}\mathrm{K} 2 \\
\mathrm{~K} 3\end{array}$ & $\begin{array}{r}136 \\
36\end{array}$ & $\begin{array}{l}11 \\
42\end{array}$ & & $\begin{array}{c}T \\
0,28\end{array}$ & & & & & & & & $\begin{array}{l}\text { K2 } \\
\text { K3 }\end{array}$ & $\begin{array}{c}322 \\
55\end{array}$ & $\begin{array}{c}4 \\
26\end{array}$ & & $\begin{array}{c}\mathrm{T} \\
-0,78\end{array}$ & \\
\hline CA54a & K1 & 156 & 2 & 1,02 & 1,28 & 1,31 & K1 & 43 & 1 & 1,01 & 1,36 & 1,37 & K1 & 241 & 24 & 1,09 & 1,02 & 1,11 \\
\hline baked & K2 & 246 & 11 & & $T$ & & K2 & 313 & 1 & & $\mathrm{~T}$ & & K2 & 138 & 26 & & $\mathrm{~T}$ & \\
\hline clay & $\mathrm{K} 3$ & 57 & 79 & & 0,88 & & K3 & 178 & 88 & & 0,94 & & K3 & 8 & 52 & & $-0,90$ & \\
\hline $\mathrm{D} 174 \mathrm{~g}$ & K1 & 152 & 19 & 1,07 & 1,07 & 1,15 & K1 & 134 & 24 & 1,04 & 1,16 & 1,21 & K1 & 152 & 62 & 1,01 & 1,04 & 1,05 \\
\hline $\begin{array}{c}\text { baked } \\
\text { clay }\end{array}$ & $\begin{array}{l}\mathrm{K} 2 \\
\mathrm{~K} 3\end{array}$ & $\begin{array}{c}57 \\
290\end{array}$ & $\begin{array}{l}15 \\
66\end{array}$ & & $\begin{array}{c}T \\
0,01\end{array}$ & & $\begin{array}{l}\text { K2 } \\
\text { K3 }\end{array}$ & $\begin{array}{c}39 \\
283\end{array}$ & $\begin{array}{l}13 \\
63\end{array}$ & & $\begin{array}{c}\mathrm{T} \\
0,57\end{array}$ & & $\begin{array}{l}\text { K2 } \\
\text { K3 }\end{array}$ & $\begin{array}{c}270 \\
5\end{array}$ & $\begin{array}{l}13 \\
23\end{array}$ & & $\begin{array}{c}T \\
0,68\end{array}$ & \\
\hline $\begin{array}{l}\text { D192v } \\
\text { baked }\end{array}$ & $\begin{array}{l}\mathrm{K} 1 \\
\mathrm{~K} 2\end{array}$ & $\begin{array}{r}65 \\
155\end{array}$ & $\begin{array}{c}14 \\
0\end{array}$ & 1,02 & $\begin{array}{c}1,06 \\
T\end{array}$ & 1,08 & & & & & & & $\begin{array}{l}\text { K1 } \\
\text { K2 }\end{array}$ & $\begin{array}{c}0 \\
117\end{array}$ & $\begin{array}{l}46 \\
23\end{array}$ & 1,05 & $\begin{array}{c}1,04 \\
T\end{array}$ & 1,09 \\
\hline clay & K3 & 242 & 76 & & 0,49 & & & & & & & & K3 & 224 & 34 & & $-0,08$ & \\
\hline $\mathrm{Hd}$ & K1 & 204 & 46 & 1,02 & 1,09 & 1,10 & K1 & 357 & 45 & 1,07 & 1,06 & 1,14 & K1 & 276 & 67 & 1,06 & 1,10 & 1,16 \\
\hline $\begin{array}{l}\text { burnt } \\
\text { soil }\end{array}$ & $\begin{array}{l}\mathrm{K} 2 \\
\mathrm{~K} 3\end{array}$ & $\begin{array}{r}26 \\
295\end{array}$ & $\begin{array}{c}44 \\
1\end{array}$ & & $\begin{array}{c}\top \\
0,67\end{array}$ & & $\begin{array}{l}\text { K2 } \\
\text { K3 }\end{array}$ & $\begin{array}{l}123 \\
233\end{array}$ & $\begin{array}{l}30 \\
29\end{array}$ & & $\begin{array}{c}\top \\
-0,10\end{array}$ & & $\begin{array}{l}\mathrm{K} 2 \\
\mathrm{~K} 3\end{array}$ & $\begin{array}{c}155 \\
61\end{array}$ & $\begin{array}{l}11 \\
18\end{array}$ & & $\begin{array}{c}T \\
0,22\end{array}$ & \\
\hline $\begin{array}{c}2073 a \\
\text { burnt }\end{array}$ & $\begin{array}{l}\mathrm{K} 1 \\
\mathrm{~K} 2\end{array}$ & $\begin{array}{l}261 \\
353\end{array}$ & $\begin{array}{c}43 \\
2\end{array}$ & 1,13 & $\begin{array}{c}1,16 \\
T\end{array}$ & 1,30 & $\begin{array}{l}\mathrm{K} 1 \\
\mathrm{~K} 2\end{array}$ & $\begin{array}{l}260 \\
357\end{array}$ & $\begin{array}{l}15 \\
24\end{array}$ & 1,01 & $\begin{array}{c}1,06 \\
T\end{array}$ & 1,07 & $\begin{array}{l}\text { K1 } \\
\text { K2 }\end{array}$ & $\begin{array}{l}233 \\
332\end{array}$ & $\begin{array}{l}26 \\
18\end{array}$ & 1,03 & $\begin{array}{c}1,01 \\
T\end{array}$ & 1,04 \\
\hline soil & K3 & 85 & 47 & & 0,10 & & $\mathrm{~K} 3$ & 141 & 61 & & 0,71 & & K3 & 93 & 57 & & $-0,69$ & \\
\hline $2085 v$ & K1 & 79 & 51 & 1,11 & 1,15 & 1,28 & K1 & 54 & 4 & 1,06 & 1,06 & 1,13 & K1 & 209 & 37 & 1,02 & 1,09 & 1,11 \\
\hline burnt & K2 & 195 & 20 & & $T$ & & K2 & 152 & 63 & & $\mathrm{~T}$ & & K2 & 87 & 34 & & $\mathrm{~T}$ & \\
\hline soil & K3 & 299 & 32 & & 0,14 & & K3 & 323 & 27 & & $-0,07$ & & K3 & 330 & 33 & & 0,62 & \\
\hline $2085 \mathrm{~g}$ & K1 & 112 & 64 & 1,09 & 1,06 & 1,16 & & & & & & & K1 & 178 & 38 & 1,03 & 1,12 & 1,16 \\
\hline burnt & K2 & 224 & 10 & & $\mathrm{~T}$ & & & & & & & & K2 & 56 & 34 & & $\mathrm{~T}$ & \\
\hline soil & K3 & 313 & 24 & & $-0,18$ & & & & & & & & K3 & 300 & 33 & & 0,60 & \\
\hline $2168 \mathrm{a}$ & K1 & 108 & 32 & 1,01 & 1,16 & 1,17 & K1 & 104 & 9 & 1,05 & 1,08 & 1,13 & K1 & 165 & 54 & 1,01 & 1,09 & 1,10 \\
\hline burnt & K2 & 214 & 24 & & $T$ & & K2 & 202 & 38 & & $\mathrm{~T}$ & & K2 & 262 & 4 & & $\mathrm{~T}$ & \\
\hline soil & $\mathrm{K} 3$ & 334 & 48 & & 0,90 & & K3 & 3 & 51 & & 0,25 & & K3 & 355 & 35 & & 0,83 & \\
\hline $2192 a$ & K1 & 31 & 60 & 1,03 & 1,08 & 1,11 & & & & & & & K1 & 106 & 60 & 1,04 & 1,08 & 1,12 \\
\hline $\begin{array}{c}\text { baked } \\
\text { clay }\end{array}$ & $\begin{array}{l}\mathrm{K} 2 \\
\mathrm{~K} 3\end{array}$ & $\begin{array}{l}210 \\
300\end{array}$ & $\begin{array}{c}30 \\
0\end{array}$ & & $\begin{array}{c}\top \\
0,43\end{array}$ & & & & & & & & $\begin{array}{l}\mathrm{K} 2 \\
\mathrm{~K} 3\end{array}$ & $\begin{array}{l}239 \\
337\end{array}$ & $\begin{array}{l}21 \\
20\end{array}$ & & $\begin{array}{c}T \\
0,29\end{array}$ & \\
\hline $2192 b$ & K1 & 351 & 75 & 104 & 1,16 & 120 & & & & & & & K1 & 197 & 1 & 106 & 105 & 112 \\
\hline baked & K2 & 163 & 15 & & $T$ & & & & & & & & K2 & 288 & 33 & & $T$ & \\
\hline clay & K3 & 254 & 2 & & 0,61 & & & & & & & & K3 & 105 & 56 & & $-0,10$ & \\
\hline DG23e & K1 & 212 & 13 & 1,02 & 1,03 & 1,05 & K1 & 296 & 28 & 1,01 & 1,07 & 1,08 & K1 & 67 & 12 & 1,02 & 1,05 & 1,08 \\
\hline $\begin{array}{c}\text { baked } \\
\text { clay }\end{array}$ & $\begin{array}{l}\mathrm{K} 2 \\
\mathrm{~K} 3\end{array}$ & $\begin{array}{l}315 \\
110\end{array}$ & $\begin{array}{l}45 \\
42\end{array}$ & & $\begin{array}{c}T \\
0,20\end{array}$ & & $\begin{array}{l}\text { K2 } \\
\text { K3 }\end{array}$ & $\begin{array}{c}31 \\
138\end{array}$ & $\begin{array}{c}9 \\
60\end{array}$ & & $\begin{array}{c}\mathrm{T} \\
0,79\end{array}$ & & $\begin{array}{l}\text { K2 } \\
\text { K3 }\end{array}$ & $\begin{array}{l}303 \\
161\end{array}$ & $\begin{array}{l}69 \\
16\end{array}$ & & $\begin{array}{c}\mathrm{T} \\
0,39\end{array}$ & \\
\hline $\begin{array}{l}\text { DG37d } \\
\text { baked }\end{array}$ & $\begin{array}{l}\mathrm{K} 1 \\
\mathrm{~K} 2\end{array}$ & $\begin{array}{c}140 \\
33\end{array}$ & $\begin{array}{l}19 \\
41\end{array}$ & 1,10 & $\begin{array}{c}1,04 \\
T\end{array}$ & 1,14 & & & & & & & $\begin{array}{l}\text { K1 } \\
\text { K2 }\end{array}$ & $\begin{array}{l}327 \\
104\end{array}$ & $\begin{array}{l}42 \\
39\end{array}$ & 1,07 & $\begin{array}{c}1,02 \\
T\end{array}$ & 1,09 \\
\hline clay & K3 & 248 & 43 & & $-0,41$ & & & & & & & & K3 & 215 & 23 & & $-0,55$ & \\
\hline DG45d & $\mathrm{K} 1$ & 110 & 28 & 1,03 & 1,08 & 1,12 & & & & & & & K1 & 131 & 41 & 1,02 & 1,05 & 1,07 \\
\hline baked & K2 & 1 & 31 & & $\mathrm{~T}$ & & & & & & & & $\mathrm{~K} 2$ & 25 & 18 & & $\mathrm{~T}$ & \\
\hline clay & $\mathrm{K} 3$ & 233 & 46 & & 0,49 & & & & & & & & K3 & 276 & 43 & & 0,40 & \\
\hline $\begin{array}{c}2241 a \\
\text { burnt }\end{array}$ & $\begin{array}{l}\mathrm{K} 1 \\
\mathrm{~K} 2\end{array}$ & $\begin{array}{c}173 \\
83\end{array}$ & $\begin{array}{c}1 \\
23\end{array}$ & 1,05 & $\begin{array}{c}1,04 \\
T\end{array}$ & 1,09 & $\begin{array}{l}\text { K1 } \\
\text { K2 }\end{array}$ & $\begin{array}{l}139 \\
229\end{array}$ & $\begin{array}{c}15 \\
2\end{array}$ & 1,01 & $\begin{array}{c}1,11 \\
T\end{array}$ & 1,12 & $\begin{array}{l}\text { K1 } \\
\text { K2 }\end{array}$ & $\begin{array}{c}119 \\
25\end{array}$ & $\begin{array}{c}6 \\
33\end{array}$ & 1,04 & $\begin{array}{c}1,11 \\
T\end{array}$ & 1,16 \\
\hline soil & $\mathrm{K} 3$ & 266 & 67 & & $-0,08$ & & K3 & 326 & 75 & & 0,75 & & K3 & 219 & 56 & & 0,42 & \\
\hline $2242 a$ & K1 & 54 & 22 & 1,04 & 1,16 & 1,21 & K1 & 91 & 38 & 1,15 & 1,43 & 1,65 & K1 & 88 & 14 & 1,12 & 1,04 & 1,16 \\
\hline burnt & K2 & 150 & 15 & & $\mathrm{~T}$ & & K2 & 181 & 1 & & $\mathrm{~T}$ & & $\mathrm{~K} 2$ & 191 & 40 & & $T$ & \\
\hline soil & $\mathrm{K} 3$ & 272 & 63 & & 0,56 & & $\mathrm{~K} 3$ & 272 & 52 & & 0,44 & & $\mathrm{~K} 3$ & 343 & 45 & & $-0,50$ & \\
\hline
\end{tabular}

ture.

The fact that sample 45-16P from the group of $27 \mathrm{BR}-\mathrm{TL}$ for which the two evaluations are made using a good holder has given a bad comparison of the directions of the principal axes (Fig. 2(b)) shows the prevailing effect of the insuffi- cient determinism of the tensor through three perpendicular measurements than that of the holder not permitting a very precise positioning. Moreover, the $P$ of this sample is not as high $\left(1.23\right.$ at $440^{\circ} \mathrm{C}$ and 1.12 at $\left.620^{\circ} \mathrm{C}\right)$. Samples with a weaker degree of anisotropy do not give satisfactory results 
Table 1. (continued).

\begin{tabular}{|c|c|c|c|c|c|c|c|c|c|c|c|c|c|c|c|c|c|c|}
\hline \multirow{3}{*}{$\begin{array}{c}\text { No } \\
\text { material }\end{array}$} & \multicolumn{6}{|c|}{ ANISOTROPY OF TRM1 } & \multicolumn{6}{|c|}{ ANISOTROPY OF TRM2 } & \multicolumn{5}{|c|}{ ANISOTROPY OF IRM } & \multirow[b]{2}{*}{$\mathrm{P}$} \\
\hline & \multicolumn{3}{|c|}{428 (deg. C) } & \multirow{2}{*}{$\begin{array}{c}\mathrm{L} \\
\mathrm{K} 1 / \mathrm{K} 2 \\
\end{array}$} & \multirow{2}{*}{$\begin{array}{c}\mathrm{F} \\
\mathrm{K} 2 / \mathrm{K} 3\end{array}$} & \multirow{2}{*}{$\begin{array}{c}\mathrm{P} \\
\mathrm{K} 1 / \mathrm{K} 3 \\
\end{array}$} & \multicolumn{3}{|c|}{550 (deg. C) } & \multirow{2}{*}{$\frac{\mathrm{L}}{\mathrm{K} 1 / \mathrm{K} 2}$} & \multirow{2}{*}{$\begin{array}{c}\mathrm{F} \\
\mathrm{K} 2 / \mathrm{K} 3 \\
\end{array}$} & \multirow{2}{*}{$\begin{array}{c}\mathrm{P} \\
\mathrm{K} 1 / \mathrm{K} 3 \\
\end{array}$} & \multicolumn{3}{|c|}{$(60 \mathrm{mT})$} & \multirow{2}{*}{$\begin{array}{c}\mathrm{L} \\
\mathrm{K} 1 / \mathrm{K} 2 \\
\end{array}$} & \multirow{2}{*}{$\begin{array}{c}\mathrm{F} \\
\mathrm{K} 2 / \mathrm{K} 3 \\
\end{array}$} & \\
\hline & & D & 1 & & & & & D & 1 & & & & & $\mathrm{D}$ & $\mathrm{T}$ & & & $\mathrm{K} 1 / \mathrm{K} 3$ \\
\hline $2253 a$ & $\mathrm{~K} 1$ & 131 & 3 & 1,03 & 1,06 & 1,10 & & & & & & & K1 & 319 & 11 & 1,03 & 1,06 & 1,09 \\
\hline $\begin{array}{c}\text { burnt } \\
\text { soil }\end{array}$ & $\begin{array}{l}\mathrm{K} 2 \\
\mathrm{~K} 3\end{array}$ & $\begin{array}{c}41 \\
232\end{array}$ & $\begin{array}{l}13 \\
77\end{array}$ & & $\begin{array}{c}\mathrm{T} \\
0,28\end{array}$ & & & & & & & & $\begin{array}{l}\text { K2 } \\
\text { K3 }\end{array}$ & $\begin{array}{l}227 \\
109\end{array}$ & $\begin{array}{c}6 \\
76\end{array}$ & & $\begin{array}{c}\mathrm{T} \\
0,33\end{array}$ & \\
\hline $\begin{array}{c}2264 \mathrm{v} \\
\text { burnt }\end{array}$ & $\begin{array}{l}\text { K1 } \\
\text { K2 }\end{array}$ & $\begin{array}{l}241 \\
147\end{array}$ & $\begin{array}{c}24 \\
8\end{array}$ & 1,05 & $\begin{array}{c}1,16 \\
T\end{array}$ & 1,21 & $\begin{array}{l}\mathrm{K} 1 \\
\mathrm{~K} 2\end{array}$ & $\begin{array}{c}132 \\
40\end{array}$ & $\begin{array}{c}8 \\
10\end{array}$ & 1,12 & $\begin{array}{c}1,13 \\
T\end{array}$ & 1,27 & $\begin{array}{l}\mathrm{K} 1 \\
\mathrm{~K} 2\end{array}$ & $\begin{array}{c}57 \\
321\end{array}$ & $\begin{array}{l}15 \\
20\end{array}$ & 1,08 & $\begin{array}{c}1,10 \\
T\end{array}$ & 1,18 \\
\hline soil & K3 & 41 & 65 & & 0,53 & & K3 & 261 & 77 & & 0,02 & & K3 & 181 & 63 & & 0,20 & \\
\hline GV33b & K1 & 179 & 0 & 1,02 & 1,03 & 1,05 & & & & & & & K1 & 188 & 44 & 1,03 & 1,07 & 1,10 \\
\hline $\begin{array}{c}\text { baked } \\
\text { clay }\end{array}$ & $\begin{array}{l}\mathrm{K} 2 \\
\mathrm{~K} 3\end{array}$ & $\begin{array}{r}89 \\
270\end{array}$ & $\begin{array}{l}33 \\
57\end{array}$ & & $\begin{array}{c}\mathrm{T} \\
0,13\end{array}$ & & & & & & & & $\begin{array}{l}\mathrm{K} 2 \\
\mathrm{~K} 3\end{array}$ & $\begin{array}{c}73 \\
324\end{array}$ & $\begin{array}{l}23 \\
36\end{array}$ & & $\begin{array}{c}T \\
0,46\end{array}$ & \\
\hline $\begin{array}{l}\text { GV45v } \\
\text { burnt }\end{array}$ & $\begin{array}{l}\mathrm{K} 1 \\
\mathrm{~K} 2\end{array}$ & $\begin{array}{c}124 \\
24\end{array}$ & $\begin{array}{l}40 \\
12\end{array}$ & 1,02 & $\begin{array}{c}1,21 \\
T\end{array}$ & 1,23 & $\begin{array}{l}\mathrm{K} 1 \\
\mathrm{~K} 2\end{array}$ & $\begin{array}{c}352 \\
92\end{array}$ & $\begin{array}{l}11 \\
42\end{array}$ & 1,03 & $\begin{array}{c}1,03 \\
T\end{array}$ & 1,06 & $\begin{array}{l}\text { K1 } \\
\text { K2 }\end{array}$ & $\begin{array}{l}261 \\
131\end{array}$ & $\begin{array}{l}28 \\
50\end{array}$ & 1,05 & $\begin{array}{c}1,03 \\
T\end{array}$ & 1,08 \\
\hline soil & K3 & 280 & 48 & & 0,80 & & K3 & 251 & 46 & & 0,08 & & K3 & 6 & 25 & & $-0,25$ & \\
\hline $2145 b$ & K1 & 126 & 44 & 1,11 & 1,07 & 1,19 & & & & & & & K1 & 336 & 30 & 1,01 & 1,06 & 1,07 \\
\hline burnt & K2 & 35 & 2 & & $T$ & & & & & & & & K2 & 86 & 30 & & $\mathrm{~T}$ & \\
\hline soil & K3 & 303 & 46 & & $-0,16$ & & & & & & & & K3 & 211 & 43 & & 0,80 & \\
\hline $\begin{array}{l}2215 b \\
\text { baked }\end{array}$ & $\begin{array}{l}\text { K1 } \\
\text { K2 }\end{array}$ & $\begin{array}{l}218 \\
128\end{array}$ & $\begin{array}{l}0 \\
9\end{array}$ & 1,00 & $\begin{array}{c}1,01 \\
T\end{array}$ & 1,01 & & & & & & & $\begin{array}{l}\text { K1 } \\
\text { K2 }\end{array}$ & $\begin{array}{l}133 \\
254\end{array}$ & $\begin{array}{l}58 \\
17\end{array}$ & 1,01 & $\begin{array}{c}1,08 \\
T\end{array}$ & 1,10 \\
\hline clay & K3 & 322 & 81 & & 0,86 & & & & & & & & K3 & 353 & 25 & & 0,69 & \\
\hline $2221 b$ & K1 & 237 & 44 & 1,08 & 1,03 & 1,12 & & & & & & & K1 & 256 & 2 & 1,05 & 1,01 & 1,06 \\
\hline baked & K2 & 357 & 28 & & $\mathrm{~T}$ & & & & & & & & K2 & 346 & 2 & & $T$ & \\
\hline clay & K3 & 107 & 33 & & $-0,41$ & & & & & & & & $\mathrm{~K} 3$ & 123 & 87 & & $-0,55$ & \\
\hline No & & & $\overline{\text { ISOTF }}$ & ROPY O & F TRM & & & AN & $\overline{\text { ISOTP }}$ & $\overline{R O P Y C}$ & F TRM & & & ANISOT & $\overline{\mathrm{TROP}}$ & SY OF & RM & \\
\hline material & 45 & 0 (deg. & & L & $F$ & $\mathrm{P}$ & & 0 (deg. & & L & $F$ & $\mathrm{P}$ & & $(60 \mathrm{mT})$ & & $\mathrm{L}$ & $F$ & $\mathrm{P}$ \\
\hline & & $D$ & 1 & $\mathrm{~K} 1 / \mathrm{K} 2$ & $\mathrm{~K} 2 / \mathrm{K} 3$ & $\mathrm{~K} 1 / \mathrm{K} 3$ & & D & 1 & $\mathrm{~K} 1 / \mathrm{K} 2$ & $\mathrm{~K} 2 / \mathrm{K} 3$ & $\mathrm{~K} 1 / \mathrm{K} 3$ & & $\mathrm{D}$ & 1 & $\mathrm{~K} 1 / \mathrm{K} 2$ & $\mathrm{~K} 2 / \mathrm{K} 3$ & $\mathrm{~K} 1 / \mathrm{K} 3$ \\
\hline $101 \mathrm{v}$ & K1 & 57 & 29 & 1,03 & 1,05 & 1,08 & K1 & 54 & 6 & 1,02 & 1,03 & 1,05 & K1 & 249 & 0 & 1,01 & 1,02 & 1,04 \\
\hline baked & K2 & 273 & 56 & & $T$ & & $\mathrm{~K} 2$ & 312 & 62 & & $T$ & & K2 & 339 & 65 & & $T$ & \\
\hline clay & K3 & 157 & 17 & & 0,24 & & K3 & 147 & 27 & & 0,02 & & $\mathrm{~K} 3$ & 159 & 24 & & 0,30 & \\
\hline $641 a$ & K1 & 79 & 39 & 1,04 & 1,06 & 1,11 & K1 & 303 & 49 & 1,03 & 1,01 & 1,05 & K1 & 269 & 6 & 1,03 & 1,02 & 1,05 \\
\hline burnt & K2 & 280 & 48 & & $T$ & & K2 & 57 & 20 & & $T$ & & K2 & 15 & 66 & & $T$ & \\
\hline soil & K3 & 178 & 11 & & 0,17 & & K3 & 162 & 34 & & $-0,52$ & & K3 & 177 & 22 & & $-0,11$ & \\
\hline $642 b$ & K1 & 44 & 8 & 1,02 & 1,05 & 1,07 & K1 & 243 & 15 & 1,02 & 1,10 & 1,12 & K1 & 1 & 64 & 1,06 & 1,12 & 1,18 \\
\hline $\begin{array}{c}\text { burnt } \\
\text { soil }\end{array}$ & $\begin{array}{l}\mathrm{K} 2 \\
\mathrm{~K} 3\end{array}$ & $\begin{array}{l}303 \\
141\end{array}$ & $\begin{array}{l}51 \\
38\end{array}$ & & $\begin{array}{c}\mathrm{T} \\
0,45\end{array}$ & & $\begin{array}{l}\text { K2 } \\
\text { K3 }\end{array}$ & $\begin{array}{c}1 \\
146\end{array}$ & $\begin{array}{l}60 \\
26\end{array}$ & & $\begin{array}{c}T \\
0,72\end{array}$ & & $\begin{array}{l}\mathrm{K} 2 \\
\mathrm{~K} 3\end{array}$ & $\begin{array}{l}237 \\
142\end{array}$ & $\begin{array}{l}13 \\
20\end{array}$ & & $\begin{array}{c}T \\
0,34\end{array}$ & \\
\hline $\begin{array}{c}94 \mathrm{~b} \\
\text { baked }\end{array}$ & $\mid \begin{array}{l}\mathrm{K} 1 \\
\mathrm{~K} 2\end{array}$ & $\begin{array}{c}175 \\
85\end{array}$ & $\begin{array}{c}1 \\
23\end{array}$ & 1,11 & $\begin{array}{c}1,02 \\
T\end{array}$ & 1,14 & $\begin{array}{l}\mathrm{K} 1 \\
\mathrm{~K} 2\end{array}$ & $\begin{array}{l}300 \\
204\end{array}$ & $\begin{array}{c}5 \\
52\end{array}$ & 1,02 & $\begin{array}{c}1,06 \\
T\end{array}$ & 1,08 & $\begin{array}{l}\mathrm{K} 1 \\
\mathrm{~K} 2\end{array}$ & $\begin{array}{c}99 \\
229\end{array}$ & $\begin{array}{l}21 \\
58\end{array}$ & 1,01 & $\begin{array}{c}1,05 \\
T\end{array}$ & 1,06 \\
\hline clay & K3 & 266 & 67 & & $-0,65$ & & K3 & 34 & 38 & & 0,59 & & K3 & 0 & 21 & & 0,69 & \\
\hline 1509a & K1 & 267 & 9 & 1,01 & 1,04 & 1,05 & K1 & 348 & 4 & 1,06 & 1,03 & 1,09 & K1 & 16 & 40 & 1,03 & 1,01 & 1,04 \\
\hline $\begin{array}{c}\text { burnt } \\
\text { soil }\end{array}$ & $\begin{array}{l}\text { K2 } \\
\text { K3 }\end{array}$ & $\begin{array}{c}18 \\
173\end{array}$ & $\begin{array}{l}67 \\
21\end{array}$ & & $\begin{array}{c}\mathrm{T} \\
0,73\end{array}$ & & $\begin{array}{l}\text { K2 } \\
\text { K3 }\end{array}$ & $\begin{array}{c}255 \\
83\end{array}$ & $\begin{array}{l}39 \\
51\end{array}$ & & $\begin{array}{c}\mathrm{T} \\
-0,32\end{array}$ & & $\begin{array}{l}\text { K2 } \\
\text { K3 }\end{array}$ & $\begin{array}{l}112 \\
211\end{array}$ & $\begin{array}{c}7 \\
49\end{array}$ & & $\begin{array}{c}\mathrm{T} \\
-0,39\end{array}$ & \\
\hline $\begin{array}{l}\text { 1788a } \\
\text { baked }\end{array}$ & $\begin{array}{l}\mathrm{K} 1 \\
\mathrm{~K} 2\end{array}$ & $\begin{array}{c}100 \\
10\end{array}$ & $\begin{array}{l}1 \\
3\end{array}$ & 1,03 & $\begin{array}{c}1,23 \\
T\end{array}$ & 1,27 & $\mid \begin{array}{l}\mathrm{K} 1 \\
\mathrm{~K} 2\end{array}$ & $\begin{array}{l}113 \\
204\end{array}$ & $\begin{array}{l}3 \\
0\end{array}$ & 1,01 & $\begin{array}{c}1,17 \\
T\end{array}$ & 1,18 & $\begin{array}{l}\mathrm{K} 1 \\
\mathrm{~K} 2\end{array}$ & $\begin{array}{c}89 \\
359\end{array}$ & $\begin{array}{l}0 \\
0\end{array}$ & 1,02 & $\begin{array}{c}1,14 \\
T\end{array}$ & 1,16 \\
\hline clay & K3 & 210 & 87 & & 0,76 & & K3 & 301 & 87 & & 0,91 & & $\mathrm{~K} 3$ & 214 & 89 & & 0,74 & \\
\hline $86 v$ & K1 & 101 & 4 & 1,03 & 1,03 & 1,06 & K1 & 117 & 8 & 1,02 & 1,02 & 1,04 & K1 & 278 & 7 & 1,01 & 1,02 & 1,03 \\
\hline baked & $\mathrm{K} 2$ & 192 & 11 & & $T$ & & $\mathrm{~K} 2$ & 26 & 8 & & $T$ & & $\mathrm{~K} 2$ & 187 & 10 & & $T$ & \\
\hline clay & K3 & 350 & 78 & & 0,03 & & K3 & 249 & 79 & & 0,03 & & K3 & 43 & 77 & & 0,20 & \\
\hline $2223 b$ & K1 & 115 & 25 & 1,05 & 1,10 & 1,16 & K1 & 115 & 27 & 1,07 & 1,09 & 1,17 & K1 & 3 & 5 & 1,03 & 1,00 & 1,04 \\
\hline $\begin{array}{c}\text { baked } \\
\text { clay }\end{array}$ & $\begin{array}{l}\text { K2 } \\
\text { K3 }\end{array}$ & $\begin{array}{c}22 \\
281\end{array}$ & $\begin{array}{c}5 \\
64\end{array}$ & & $\begin{array}{c}\mathrm{T} \\
0,28\end{array}$ & & $\mid \begin{array}{l}\text { K2 } \\
\text { K3 }\end{array}$ & $\begin{array}{c}16 \\
257\end{array}$ & $\begin{array}{l}17 \\
58\end{array}$ & & $\begin{array}{c}\mathrm{T} \\
0,15\end{array}$ & & $\begin{array}{l}\mathrm{K} 2 \\
\mathrm{~K} 3\end{array}$ & $\begin{array}{l}135 \\
272\end{array}$ & $\begin{array}{c}82 \\
5\end{array}$ & & $\begin{array}{c}\mathrm{T} \\
0,77\end{array}$ & \\
\hline 1576a & K1 & 53 & 45 & 1,05 & 1,12 & 1,16 & K1 & 48 & 47 & 1,11 & 1,14 & 1,27 & K1 & 55 & 58 & 1,17 & 1,10 & 1,29 \\
\hline burnt & $\mathrm{K} 2$ & 160 & 17 & & $\mathrm{~T}$ & & K2 & 158 & 18 & & $\mathrm{~T}$ & & K2 & 167 & 12 & & $\mathrm{~T}$ & \\
\hline soil & K3 & 265 & 40 & & 0,34 & & K3 & 263 & 38 & & 0,14 & & K3 & 263 & 28 & & $-0,25$ & \\
\hline
\end{tabular}

on the directions of the principal axes of the TRM ellipsoid and, thus, the tensors' orientations cannot be compared reliably. Therefore, it should be noted that for the purposes of any anisotropy correction of the palaeointensity results, the tensor determination by measurements on three perpendicular directions, as done here, is sufficient-but probably not sufficient for determination of the directions of the principal axes, especially for samples with a weak anisotropy.

The BC-BS samples do not show a good consistency among the values of anisotropy parameters (Fig. 2(c); Table 1). The degree of TRM anisotropy at two different temperature levels for the BR-TL samples is similar, as shown in Garcia (1996) and Chauvin et al. (2000), although there are some outliers here (Fig. 2(d); Table 2). The sim- 
Table 2. The same as in Table 1 but for samples from bricks and tiles (BR-TL). The lower temperature is $440^{\circ}$ or $450^{\circ} \mathrm{C}$ and the higher temperature, $550^{\circ} \mathrm{C}$ or $620^{\circ} \mathrm{C}$.

\begin{tabular}{|c|c|c|c|c|c|c|c|c|c|c|c|c|c|c|c|c|c|c|}
\hline \multirow{3}{*}{\begin{tabular}{|l|} 
No \\
material \\
\end{tabular}} & \multicolumn{6}{|c|}{ ANISOTROPY OF TRM1 } & \multicolumn{6}{|c|}{ ANISOTROPY OF TRM2 } & & \multicolumn{5}{|c|}{ ANISOTROPY OF IRM } \\
\hline & \multicolumn{3}{|c|}{450 (deg. C) } & \multirow{2}{*}{$\frac{\mathrm{L}}{\mathrm{K} 1 / \mathrm{K} 2}$} & \multirow{2}{*}{$\frac{F}{K 2 / K 3}$} & \multirow{2}{*}{$\frac{\mathrm{P}}{\mathrm{K} 1 / \mathrm{K} 3}$} & \multicolumn{3}{|c|}{550 (deg. C) } & \multirow{2}{*}{$\frac{\mathrm{L}}{\mathrm{K} 1 / \mathrm{K} 2}$} & \multirow{2}{*}{$\frac{F}{K 2 / K 3}$} & \multirow{2}{*}{$\frac{\mathrm{P}}{\mathrm{K} 1 / \mathrm{K} 3}$} & & \multicolumn{2}{|c|}{$(60 \mathrm{mT})$} & L & $F$ & $\mathrm{P}$ \\
\hline & & $\mathrm{D}$ & 1 & & & & & $\mathrm{D}$ & 1 & & & & & D & 1 & $\mathrm{~K} 1 / \mathrm{K} 2$ & $\mathrm{~K} 2 / \mathrm{K} 3$ & $\mathrm{~K} 1 / \mathrm{K} 3$ \\
\hline $69 b$ & K1 & 353 & 46 & 1.05 & 1.03 & 1.08 & K1 & 331 & 63 & 1.04 & 1.06 & 1.11 & K1 & 333 & 41 & 1.03 & 1.02 & 1.05 \\
\hline brick & K2 & 223 & 31 & & $\mathrm{~T}$ & & K2 & 207 & 15 & & $\mathrm{~T}$ & & K2 & 243 & 0 & & $\mathrm{~T}$ & \\
\hline & K3 & 115 & 27 & & -0.33 & & K3 & 111 & 21 & & 0.20 & & K3 & 152 & 48 & & -0.26 & \\
\hline $268 a$ & K1 & 170 & 1 & 1.06 & 1.18 & 1.25 & K1 & 172 & 10 & 1.06 & 1.2 & 1.27 & K1 & 344 & 7 & 1.05 & 1.11 & 1.16 \\
\hline brick & K2 & 266 & 80 & & T & & K2 & 307 & 76 & & $\mathrm{~T}$ & & K2 & 207 & 79 & & T & \\
\hline & K3 & 80 & 10 & & 0.45 & & K3 & 80 & 10 & & 0.54 & & K3 & 75 & 7 & & 0.38 & \\
\hline $269 a$ & K1 & 171 & 50 & 1.08 & 1.27 & 1.38 & K1 & 174 & 54 & 1.07 & 1.29 & 1.38 & K1 & 163 & 77 & 1.12 & 1.14 & 1.28 \\
\hline brick & K2 & 0 & 39 & & $\mathrm{~T}$ & & $\mathrm{~K} 2$ & 359 & 36 & & $\mathrm{~T}$ & & $\mathrm{~K} 2$ & 2 & 12 & & $T$ & \\
\hline & K3 & 266 & 5 & & 0.49 & & K3 & 267 & 3 & & 0.58 & & K3 & 271 & 4 & & 0.12 & \\
\hline $302 v$ & K1 & 6 & 9 & 1.05 & 1.18 & 1.25 & K1 & 5 & 11 & 1.08 & 1.19 & 1.29 & K1 & 5 & 14 & 1.03 & 1.13 & 1.17 \\
\hline brick & K2 & 249 & 70 & & $T$ & & K2 & 245 & 68 & & $T$ & & K2 & 248 & 61 & & $\mathrm{~T}$ & \\
\hline & K3 & 99 & 18 & & 0.42 & & K3 & 98 & 19 & & 0.41 & & K3 & 102 & 24 & & 0.60 & \\
\hline $376 b$ & K1 & 170 & 2 & 1.06 & 1.14 & 1.21 & K1 & 177 & 5 & 1.04 & 1.15 & 1.20 & K1 & 248 & 38 & 1.01 & 1.12 & 1.14 \\
\hline brick & K2 & 259 & 32 & & $\mathrm{~T}$ & & K2 & 271 & 37 & & $T$ & & K2 & 341 & 3 & & $\mathrm{~T}$ & \\
\hline & K3 & 83 & 58 & & 0.42 & & K3 & 81 & 53 & & 0.56 & & K3 & 76 & 51 & & 0.83 & \\
\hline $1609 a$ & K1 & 112 & 40 & 1.09 & 1.05 & 1.14 & K1 & 141 & 46 & 1.07 & 1.06 & 1.13 & K1 & 110 & 62 & 1.11 & 1.08 & 1.20 \\
\hline brick & K2 & 221 & 21 & & $T$ & & K2 & 236 & 4 & & $\mathrm{~T}$ & & K2 & 229 & 14 & & $\mathrm{~T}$ & \\
\hline & K3 & 332 & 42 & & -0.32 & & K3 & 330 & 44 & & -0.12 & & K3 & 325 & 24 & & -0.15 & \\
\hline $70 \mathrm{~b}$ & K1 & 198 & 10 & 1.06 & 1.03 & 1.09 & $\mathrm{~K} 1$ & 204 & 4 & 1.05 & 1.04 & 1.10 & K1 & 216 & 17 & 1.06 & 1.04 & 1.10 \\
\hline brick & K2 & 297 & 44 & & $\mathrm{~T}$ & & K2 & 300 & 52 & & $\mathrm{~T}$ & & $\mathrm{~K} 2$ & 346 & 63 & & $\mathrm{~T}$ & \\
\hline & K3 & 98 & 45 & & -0.25 & & K3 & 111 & 37 & & -0.15 & & K3 & 119 & 18 & & -0.19 & \\
\hline $270 a$ & K1 & 188 & 7 & 1.06 & 1.16 & 1.22 & K1 & 11 & 3 & 1.04 & 1.17 & 1.21 & K1 & 46 & 51 & 1.01 & 1.11 & 1.12 \\
\hline brick & K2 & 75 & 74 & & $\mathrm{~T}$ & & K2 & 110 & 70 & & $\mathrm{~T}$ & & K2 & 185 & 31 & & $T$ & \\
\hline & K3 & 280 & 15 & & 0.44 & & K3 & 280 & 19 & & 0.62 & & K3 & 288 & 21 & & 0.83 & \\
\hline $305 a$ & K1 & 353 & 3 & 1.10 & 1.32 & 1.46 & K1 & 353 & 10 & 1.06 & 1.33 & 1.40 & K1 & 349 & 17 & 1.04 & 1.15 & 1.20 \\
\hline brick & K2 & 120 & 85 & & $T$ & & K2 & 154 & 79 & & $\mathrm{~T}$ & & K2 & 142 & 70 & & $\mathrm{~T}$ & \\
\hline & K3 & 263 & 4 & & 0.47 & & K3 & 262 & 3 & & 0.67 & & K3 & 256 & 8 & & 0.55 & \\
\hline $379 a$ & K1 & 285 & 60 & 1.03 & 1.04 & 1.06 & K1 & 299 & 63 & 1.02 & 1.05 & 1.07 & K1 & 11 & 48 & 1.02 & 1.03 & 1.06 \\
\hline brick & K2 & 189 & 3 & & $\mathrm{~T}$ & & K2 & 196 & 6 & & $\mathrm{~T}$ & & K2 & 226 & 36 & & $T$ & \\
\hline & K3 & 98 & 30 & & 0.17 & & K3 & 102 & 26 & & 0.27 & & K3 & 122 & 17 & & 0.28 & \\
\hline $551 b$ & K1 & 39 & 2 & 1.04 & 1.03 & 1.07 & K1 & 216 & 6 & 1.02 & 1.04 & 1.07 & K1 & 236 & 1 & 1.03 & 1.02 & 1.05 \\
\hline brick & K2 & 129 & 13 & & $T$ & & K2 & 126 & 1 & & $T$ & & K2 & 326 & 7 & & $T$ & \\
\hline in constr. & K3 & 299 & 77 & & -0.08 & & K3 & 30 & 84 & & 0.33 & & K3 & 138 & 83 & & -0.26 & \\
\hline & & & & & $\mathrm{T}$ & & & & & & & & & & & & & \\
\hline $36-3$ & K1 & 274 & 68 & 1.06 & 1.05 & 1.11 & K1 & 227 & 22 & 1.04 & 1.06 & 1.11 & K1 & 249 & 54 & 1.04 & 1.02 & 1.06 \\
\hline tile & K2 & 47 & 15 & & $T$ & & K2 & 347 & 51 & & $T$ & & $\mathrm{~K} 2$ & 123 & 22 & & $\mathrm{~T}$ & \\
\hline in constr. & K3 & 141 & 15 & & -0.06 & & K3 & 124 & 30 & & 0.20 & & K3 & 21 & 25 & & -0.39 & \\
\hline $36-8$ & $\mathrm{~K} 1$ & 294 & 19 & 1.03 & 1.09 & 1.12 & K1 & 61 & 18 & 1.05 & 1.07 & 1.12 & K1 & 85 & 45 & 1.07 & 1.08 & 1.15 \\
\hline tile & K2 & 42 & 41 & & $T$ & & $\mathrm{~K} 2$ & 323 & 22 & & $T$ & & K2 & 306 & 37 & & $T$ & \\
\hline in constr. & K3 & 186 & 43 & & 0.52 & & K3 & 187 & 61 & & 0.19 & & K3 & 198 & 22 & & 0.10 & \\
\hline $36-13$ & K1 & 14 & 29 & 1.07 & 1.09 & 1.17 & $\mathrm{~K} 1$ & 2 & 21 & 1.02 & 1.14 & 1.17 & K1 & 301 & 32 & 1.02 & 1.07 & 1.10 \\
\hline tile & K2 & 107 & 6 & & $T$ & & K2 & 269 & 6 & & $T$ & & K2 & 44 & 19 & & $T$ & \\
\hline in constr. & K3 & 207 & 61 & & 0.11 & & K3 & 163 & 68 & & 0.72 & & K3 & 159 & 50 & & 0.52 & \\
\hline $36-16$ & K1 & 222 & 9 & 1.08 & 1.08 & 1.17 & K1 & 48 & 2 & 1.21 & 1.07 & 1.29 & K1 & 63 & 7 & 1.02 & 1.02 & 1.05 \\
\hline tile & K2 & 319 & 40 & & $T$ & & K2 & 302 & 81 & & $T$ & & K2 & 331 & 12 & & $T$ & \\
\hline in constr. & K3 & 121 & 49 & & 0.02 & & K3 & 138 & 9 & & -0.46 & & K3 & 182 & 76 & & -0.01 & \\
\hline $36-17$ & K1 & 210 & 11 & 1.03 & 1.01 & 1.04 & K1 & 232 & 1 & 1.08 & 1.04 & 1.12 & K1 & 257 & 9 & 1.02 & 1.07 & 1.09 \\
\hline tile & K2 & 114 & 29 & & $\mathrm{~T}$ & & K2 & 322 & 10 & & $\mathrm{~T}$ & & K2 & 163 & 21 & & $T$ & \\
\hline in consrt. & K3 & 318 & 59 & & -0.61 & & K3 & 134 & 80 & & -0.36 & & K3 & 9 & 66 & & 0.39 & \\
\hline & & & & & & & & & & & & & & & & & & \\
\hline
\end{tabular}

ple recalculation of the regression line without these out- but pointing that their influence is not as important. liers gives a similar regression equation: $P($ TRM2) $=$ Nevertheless, we have strong proof that the anisotropy $0.9386 P($ TRM1 $)+0.0778$, with a significantly better re- evaluations at two temperatures are in general identicalgression coefficient $\left(R^{2}=0.8776\right)$ as should be expected, as is shown in Fig. 3, where the relationship between the 
Table 2. (continued).

\begin{tabular}{|c|c|c|c|c|c|c|c|c|c|c|c|c|c|c|c|c|c|c|}
\hline \multirow{3}{*}{\begin{tabular}{|l|} 
No \\
material \\
\end{tabular}} & \multicolumn{6}{|c|}{ ANISOTROPY OF TRM1 } & \multicolumn{6}{|c|}{ ANISOTROPY OF TRM2 } & & \multicolumn{5}{|c|}{ ANISOTROPY OF IRM } \\
\hline & \multicolumn{3}{|c|}{450 (deg. C) } & \multirow{2}{*}{$\frac{\mathrm{L}}{\mathrm{K} 1 / \mathrm{K} 2}$} & \multirow{2}{*}{$\frac{F}{K 2 / K 3}$} & \multirow{2}{*}{$\frac{\mathrm{P}}{\mathrm{K} 1 / \mathrm{K} 3}$} & \multicolumn{3}{|c|}{550 (deg. C) } & \multirow{2}{*}{\multicolumn{2}{|c|}{$\begin{array}{cc}\mathrm{L} & \mathrm{F} \\
\mathrm{K} 1 / \mathrm{K} 2 & \mathrm{~K} 2 / \mathrm{K} 3 \\
\end{array}$}} & \multirow{2}{*}{$\frac{\mathrm{P}}{\mathrm{K} 1 / \mathrm{K} 3}$} & & \multicolumn{2}{|c|}{ (60 mT) } & \multirow{2}{*}{$\frac{\mathrm{L}}{\mathrm{K} 1 / \mathrm{K} 2}$} & $\mathrm{~F}$ & $\mathrm{P}$ \\
\hline & & D & $\mathrm{I}$ & & & & & D & 1 & & & & & D & 1 & & $\mathrm{~K} 2 / \mathrm{K} 3$ & $\mathrm{~K} 1 / \mathrm{K} 3$ \\
\hline $36-19$ & $\mathrm{~K} 1$ & 153 & 9 & 1.07 & 1.03 & 1.10 & K1 & 135 & 14 & 1.08 & 1.02 & 1.11 & K1 & 114 & 9 & 1.02 & 1.03 & 1.04 \\
\hline tile & K2 & 289 & 78 & & $\mathrm{~T}$ & & K2 & 35 & 35 & & $\mathrm{~T}$ & & K2 & 268 & 79 & & $T$ & \\
\hline in constr. & K3 & 61 & 8 & & -0.40 & & K3 & 243 & 51 & & -0.54 & & K3 & 24 & 4 & & 0.25 & \\
\hline $41-1$ & $\mathrm{~K} 1$ & 48 & 10 & 1.18 & 1.05 & 1.23 & $\mathrm{~K} 1$ & 32 & 3 & 1.13 & 1.10 & 1.23 & K1 & 294 & 1 & 1.14 & 1.06 & 1.20 \\
\hline tile & K2 & 151 & 50 & & $\mathrm{~T}$ & & K2 & 122 & 2 & & $\mathrm{~T}$ & & K2 & 31 & 85 & & $\mathrm{~T}$ & \\
\hline in constr. & $\mathrm{K} 3$ & 310 & 38 & & -0.57 & & K3 & 250 & 86 & & -0.13 & & K3 & 204 & 5 & & -0.37 & \\
\hline $41-4$ & K1 & 52 & 12 & 1.07 & 1.08 & 1.16 & K1 & 155 & 3 & 1.13 & 1.10 & 1.24 & K1 & 102 & 7 & 1.02 & 1.11 & 1.14 \\
\hline tile & $\mathrm{K} 2$ & 146 & 17 & & $\mathrm{~T}$ & & K2 & 64 & 7 & & $T$ & & K2 & 193 & 7 & & $T$ & \\
\hline in constr. & K3 & 288 & 69 & & 0.04 & & K3 & 272 & 83 & & -0.16 & & K3 & 329 & 79 & & 0.75 & \\
\hline $41-6$ & $\mathrm{~K} 1$ & 53 & 2 & 1.05 & 1.09 & 1.14 & K1 & 160 & 8 & 1.08 & 1.04 & 1.12 & K1 & 247 & 1 & 1.01 & 1.04 & 1.04 \\
\hline tile & K2 & 151 & 79 & & $\mathrm{~T}$ & & $\mathrm{~K} 2$ & 252 & 45 & & $\mathrm{~T}$ & & K2 & 337 & 0 & & $T$ & \\
\hline in constr. & K3 & 322 & 11 & & 0.24 & & K3 & 36 & 75 & & -0.34 & & K3 & 85 & 88 & & 0.70 & \\
\hline $41-8$ & K1 & 225 & 16 & 1.11 & 1.06 & 1.18 & K1 & 89 & 1 & 1.06 & 1.05 & 1.12 & K1 & 240 & 37 & 1.01 & 1.01 & 1.02 \\
\hline tile & $\mathrm{K} 2$ & 48 & 74 & & $T$ & & K2 & 181 & 62 & & $\mathrm{~T}$ & & K2 & 51 & 52 & & $\mathrm{~T}$ & \\
\hline in constr. & K3 & 315 & 1 & & -0.26 & & K3 & 91 & 0 & & -0.05 & & K3 & 147 & 4 & & 0.17 & \\
\hline $41-9$ & K1 & 157 & 0 & 1.07 & 1.14 & 1.22 & $\mathrm{~K} 1$ & 33 & 8 & 1.09 & 1.10 & 1.19 & K1 & 53 & 13 & 1.02 & 1.07 & 1.09 \\
\hline tile & K2 & 67 & 2 & & $\mathrm{~T}$ & & K2 & 124 & 3 & & $T$ & & K2 & 320 & 10 & & $T$ & \\
\hline in constr. & $\mathrm{K} 3$ & 257 & 88 & & 0.28 & & K3 & 235 & 81 & & 0.04 & & K3 & 193 & 73 & & 0.50 & \\
\hline $41-19$ & K1 & 25 & 30 & 1.10 & 1.04 & 1.15 & K1 & 304 & 13 & 1.05 & 1.05 & 1.10 & K1 & 251 & 11 & 1.02 & 1.04 & 1.06 \\
\hline tile & $\mathrm{K} 2$ & 258 & 47 & & $\mathrm{~T}$ & & K2 & 36 & 9 & & $T$ & & K2 & 348 & 29 & & $T$ & \\
\hline in constr. & $\mathrm{K} 3$ & 133 & 28 & & -0.36 & & K3 & 159 & 74 & & -0.06 & & K3 & 143 & 57 & & 0.38 & \\
\hline & & ANISC & $\overline{\text { TTRC }}$ & DPY OF & F TRM & & & ANISC & $\overline{\text { TRRC }}$ & DPY OF & $=\mathrm{TRM}$ & & & & & & & \\
\hline material & 440 & 0 (deg. $C$ & & $\mathrm{~L}$ & $\mathrm{~F}$ & $\mathrm{P}$ & 620 & (deg. & & L & F & $\mathrm{P}$ & & & & & & \\
\hline & & $\mathrm{D}$ & 1 & $\mathrm{~K} 1 / \mathrm{K} 2$ & $\mathrm{~K} 2 / \mathrm{K} 3$ & $\mathrm{~K} 1 / \mathrm{K} 3$ & & $\mathrm{D}$ & 1 & $\mathrm{~K} 1 / \mathrm{K} 2$ & $\mathrm{~K} 2 / \mathrm{K} 3$ & $\mathrm{~K} 1 / \mathrm{K} 3$ & & & & & & \\
\hline $41-6 a$ & $\mathrm{~K} 1$ & 86 & 11 & 1.01 & 1.07 & 1.08 & K1 & 110 & 15 & 1.00 & 1.10 & 1.10 & & & & & & \\
\hline tile & $\mathrm{K} 2$ & 179 & 14 & & $T$ & & K2 & 202 & 6 & & $T$ & & & & & & & \\
\hline in constr. & K3 & 319 & 72 & & 0.73 & & K3 & 313 & 74 & & 0.92 & & & & & & & \\
\hline $41-19 a$ & K1 & 215 & 8 & 1.03 & 1.03 & 1.07 & K1 & 327 & 13 & 1.07 & 1.04 & 1.11 & & & & & & \\
\hline tile & K2 & 315 & 52 & & $\mathrm{~T}$ & & K2 & 236 & 6 & & $\mathrm{~T}$ & & & & & & & \\
\hline in constr. & K3 & 119 & 37 & & -0.06 & & K3 & 121 & 76 & & -0.24 & & & & & & & \\
\hline $\mathrm{B} 175 \mathrm{~b}$ & K1 & 207 & 50 & 1.02 & 1.01 & 1.03 & K1 & 196 & 20 & 1.02 & 1.01 & 1.03 & & & & & & \\
\hline brick & $\mathrm{K} 2$ & 6 & 38 & & $T$ & & K2 & 98 & 25 & & $T$ & & & & & & & \\
\hline & K3 & 104 & 11 & & -0.21 & & K3 & 323 & 57 & & -0.47 & & & & & & & \\
\hline B178a & K1 & 193 & 29 & 1.06 & 1.07 & 1.13 & K1 & 180 & 27 & 1.04 & 1.05 & 1.09 & & & & & & \\
\hline brick & $\mathrm{K} 2$ & 84 & 30 & & $\mathrm{~T}$ & & $\mathrm{~K} 2$ & 72 & 32 & & $T$ & & & & & & & \\
\hline & K3 & 318 & 46 & & 0.10 & & K3 & 302 & 46 & & 0.03 & & & & & & & \\
\hline B182b & $\mathrm{K} 1$ & 184 & 62 & 1.06 & 1.05 & 1.12 & K1 & 313 & 61 & 1.03 & 1.05 & 1.07 & & & & & & \\
\hline brick & K2 & 338 & 26 & & $T$ & & K2 & 177 & 22 & & $T$ & & & & & & & \\
\hline & K3 & 73 & 11 & & -0.07 & & $\mathrm{~K} 3$ & 79 & 18 & & 0.27 & & & & & & & \\
\hline B207b & $\mathrm{K} 1$ & 153 & 20 & 1.05 & 1.02 & 1.07 & K1 & 157 & 25 & 1.05 & 1.00 & 1.06 & & & & & & \\
\hline brick & K2 & 60 & 9 & & $\mathrm{~T}$ & & K2 & 264 & 32 & & $T$ & & & & & & & \\
\hline & $\mathrm{K} 3$ & 307 & 68 & & -0.33 & & K3 & 37 & 47 & & -0.94 & & & & & & & \\
\hline B210a & K1 & 248 & 58 & 1.03 & 1.04 & 107 & K1 & 250 & 64 & 1.05 & 1.03 & 1.08 & & & & & & \\
\hline brick & $\mathrm{K} 2$ & 359 & 12 & & $T$ & & $\mathrm{~K} 2$ & 9 & 13 & & $T$ & & & & & & & \\
\hline & K3 & 95 & 28 & & 0.12 & & K3 & 104 & 22 & & -0.28 & & & & & & & \\
\hline B211a & K1 & 329 & 3 & 1.02 & 1.02 & 1.04 & K1 & 205 & 3 & 1.00 & 1.04 & 1.04 & & & & & & \\
\hline brick & $\mathrm{K} 2$ & 233 & 59 & & $\mathrm{~T}$ & & K2 & 256 & 76 & & $\mathrm{~T}$ & & & & & & & \\
\hline & $\mathrm{K} 3$ & 61 & 31 & & 0.08 & & K3 & 64 & 14 & & 0.90 & & & & & & & \\
\hline & & & & & & & & & & & & & & & & & & \\
\hline & & & & & & & & & & & & & & & & & & \\
\hline
\end{tabular}

normalized principal values of TRM1 and TRM2 ellipsoids (normalization made by their average) for all samples subjected to two evaluations is given. In the case when a specially designed holder was used for the two evaluations
(Fig. 3(b)), the linear relation is clearly close to 1 , pointing to the origin of the system of co-ordinates and thus proving the identical shape of the anisotropy ellipsoids (Stephenson, 1986; Hus et al., 2002). The results of samples for which a 
Table 2. (continued).

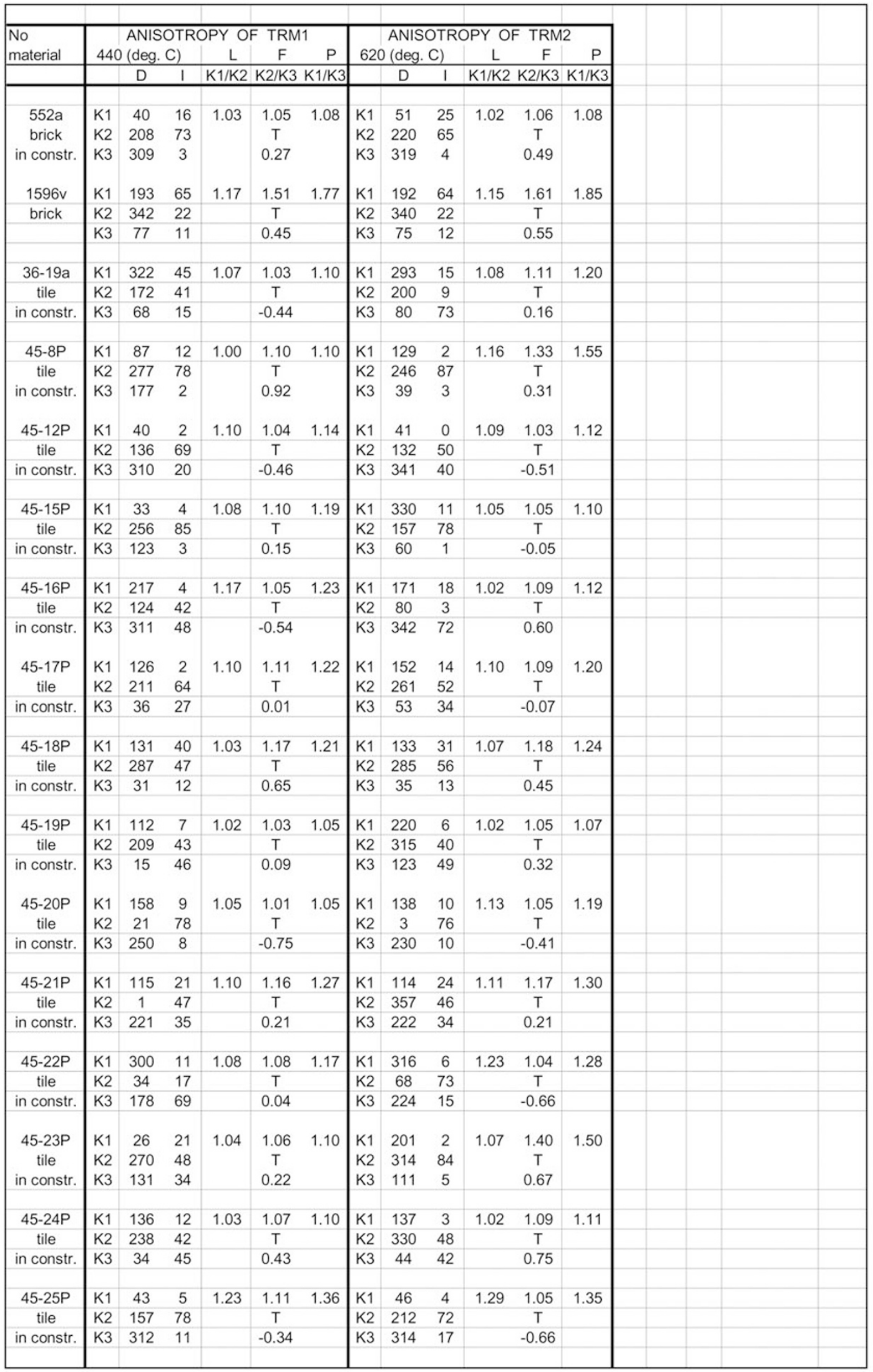

doubt exists on the positioning at lower temperatures show a slope quite close to 1 but statistically different from 1 (1 does not enter the confidence interval 0.9695-0.8857, taking into account the standard error of the regression coeffi- cient). The same concerns the intercept ( 0 is not included in the confidence interval $0.0724 \pm 0.042$ ). Evidently this is due to the observed deviations of $q$-min and $q$-max mostly for weaker anisotropic BC-BS samples (Fig. 3(a)). 
Table 2. (continued).

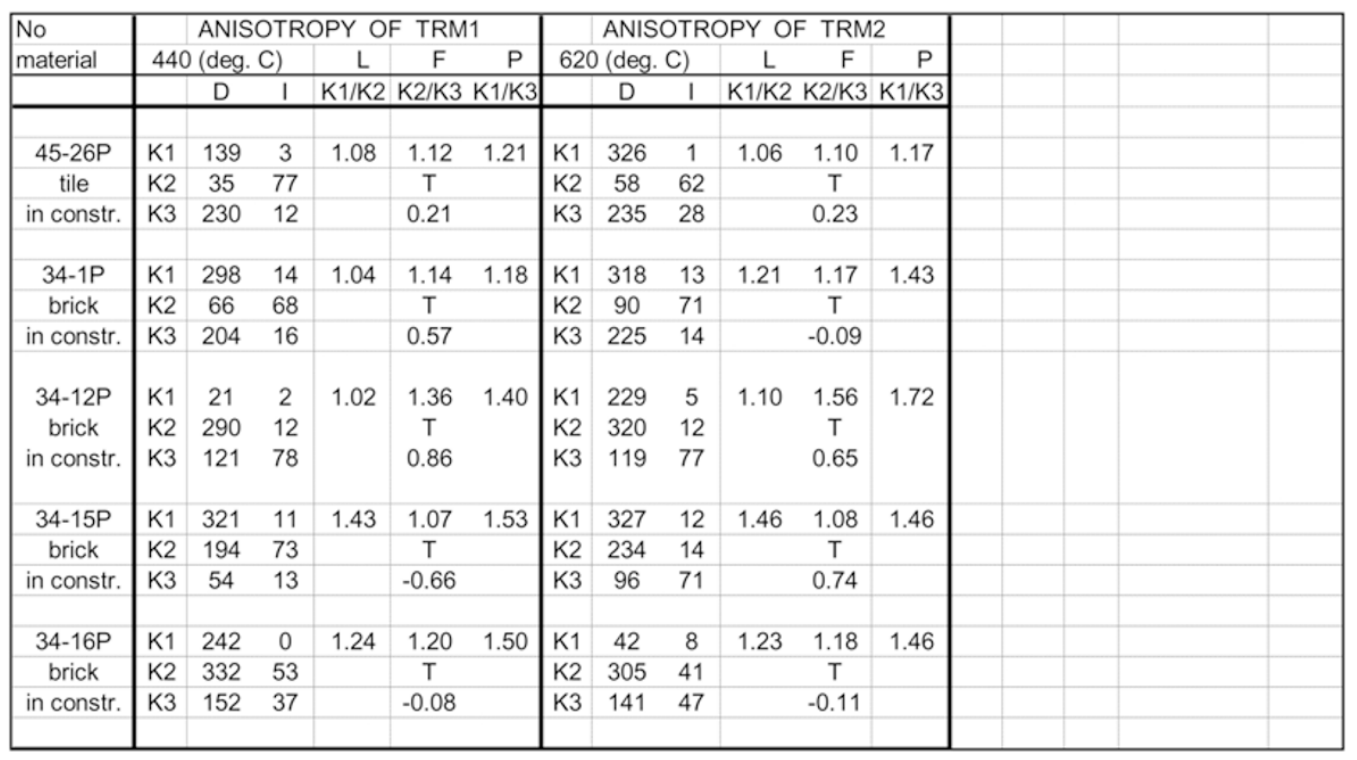

The shape parameter $T$ for different temperatures and different kinds of samples is given in Fig. 4. Obviously, the oblate form of anisotropy ellipsoids prevails for all kind of samples and for the two evaluations. This is linked with the shape-related magnetic fabric for bricks and tiles and with the mechanically produced one for baked clay ovens' floors. The similarity in the TRM anisotropy evaluated at two temperatures is even better revealed in case of pottery having the highly expressed 'easy plane' of magnetization (Genevey et al., 2003).

\subsection{Comparison of TRM and IRM anisotropy param- eters}

The IRM anisotropy evaluation of the 24 prehistoric samples was carried out in the Sofia laboratory after the Thellier experiment had been performed in the Rennes laboratory. The IRM anisotropy determination for the other 32 samples of different type and age was also carried out in the Rennes laboratory after the Thellier experiment. The only difference is that the residuals are measured after the subsequent three-axis AF static demagnetization in Rennes. There are 29 BR-TL samples for which the IRM anisotropy had not been determined. We will therefore compare IRM and TRM anisotropy tensors for 56 samples altogether.

We have shown that, in the case of TRM tensors obtained for two temperatures, the directions of the principal axes do not coincide very well in case of lower anisotropy of baked clay plasters and burnt soils. Because of this weak anisotropy and related large confidence angles (see Fig. 2(b)), mutual permutation of the principal axes is possible. This may be a reason for the moderate correlation that was obtained between anisotropy parameters of IRM and TRM1 or TRM2 tensors (Fig. 5(a, c)). The expected linear dependence between $P($ IRM $)$ and $P($ TRM) is badly expressed $\left(r^{2}=0.21\right.$ for Fig. 5(a) and $r^{2}=0.4$ for Fig. 5(c)) for the two temperatures, with a slight amelioration when a better holder was used for the TRM2 anisotropy measurements. Without always being the case, the remanence fabric in the studied collection of archaeological materials points to prevailed flattening for both BC-BS and BR-TL
(Fig. 5(b, d)).

The normalized principal values of the IRM $\left(r_{i}\right)$ and TRM $\left(q_{i}\right)$ ellipsoids (the normalization also made by their average) for all studied samples show a good linear relationship for each of the two TRM1 (Fig. 6(a)) and TRM2 (Fig. 7(a)) anisotropy evaluations. Such a relationship can be considered to be a precondition for a similar shape of both tensors, as shown by Stephenson et al. (1986) when the regression line passes through the origin of the coordinate system. The linear relationship between $r_{i}$ and $q_{i}$ in our case deviates from the expected slope (not crossing the origin), which points to different shapes of the two ellipsoids (Figs. 6(a) and 7(a)). If we consider the same relationship separately for BC-BS and for BR-TL, we come to an interesting conclusion (Figs. 6(b, c) and 7(b, c)). It appears that the regression line of normalized principal values of BC-BS samples obtained for the two temperatures (Figs. 6(b) and 7(b)) deviates negligibly from the origin of the coordinate system (statistically, 1 and 0 enter the confidence intervals of the regression coefficient and of the intercept), suggesting the similarity of the two ellipsoid shapes. In this case, we have a confirmation of the Stephenson et al. (1986) assumption for the equality of TRM and IRM anisotropy ellipsoids shapes made in these authors' paper on the basis of only five samples. The increase in the correlation coefficient for BC-BS at the second anisotropy evaluation (TRM2; Fig. 7(b)) reflects the usage of better sample holders in this case. The regression line between the $r_{i}$ and $q_{i}$ obtained from BR-TL samples (Figs. 6(c) and 7(c)) with a good correlation coefficient $\left(R^{2}=0.85\right.$ and $\left.R^{2}=0.83\right)$ deviates substantially from the origin of the coordinate system. This observation indicates that our results obtained from bricks/tile samples do not confirm the equality of the TRM and IRM ellipsoid shapes.

\section{Anisotropy Effect on the Palaeointensity Eval- uations}

In further studies we used the TRM1 anisotropy parameters obtained at intermediate temperatures of 428,440 , or 


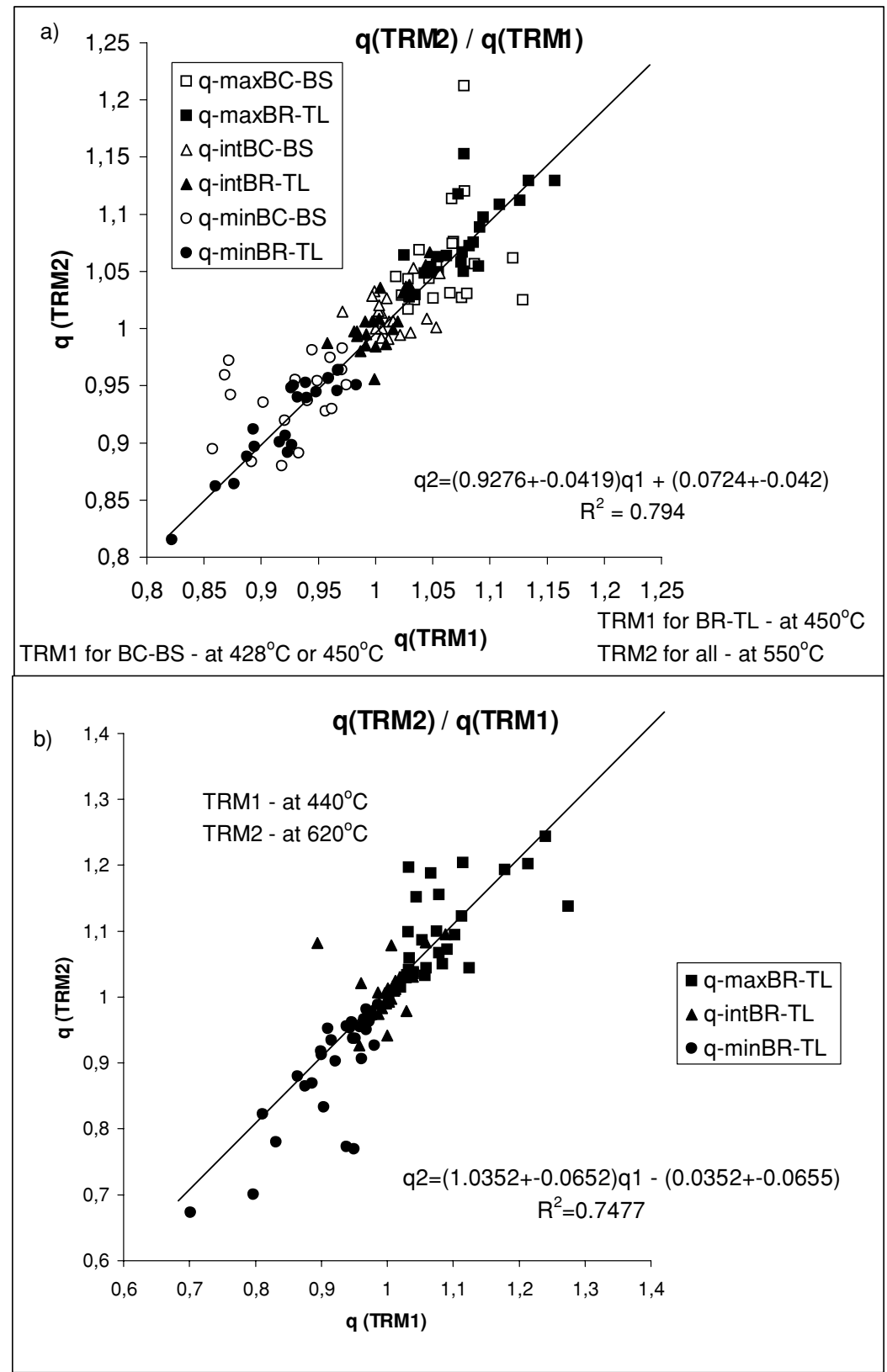

Fig. 3. Relationship between normalized principal values of the $\operatorname{TRM} 1(q 1)$ and $\operatorname{TRM} 2(q 2)$ ellipsoids measured at two temperatures $\left(q_{x}=\mathrm{TRM}_{x x} /\left(\left(\mathrm{TRM}_{x x}+\mathrm{TRM}_{y y}+\mathrm{TRM}_{z z}\right) / 3\right)\right.$ etc.) for the studied samples: (a) results from several experimental series for palaeointensity determination are combined; (b) results from a large BR-TL series for palaeointensity determination with two TRM anisotropy evaluations using a holder permitting good positioning of the samples. Temperature levels are given correspondingly. The best fitted straight lines are given with their errors.

$450^{\circ} \mathrm{C}$, paying particular attention to the identical shapes of the anisotropy ellipsoids obtained by the evaluations at two temperatures (Fig. 3(b)). Moreover and very important, these steps were included in the temperature interval for the palaeointensity determinations when the experimental points of $550^{\circ}$ or $620^{\circ} \mathrm{C}$ at which the TRM2 anisotropy had been evaluated were only rarely taken for calculation of palaeointensity results. Some examples are given in Fig. 8.

The degree of anisotropy $P(\mathrm{TRM}), P(\mathrm{IRM})$ and the correction factors $f$ determined on each sample, using both TRM and IRM tensors and the direction of NRM, in sample co-ordinates, are shown in Table 3 . In this table, the obtained correction factor $f_{\mathrm{IRM}}$ for BR-TL, using the IRM anisotropy ellipsoid, are given in italics, and they are not taken for further considerations. The histogram of correction factors $f$ obtained through TRM and IRM anisotropy determinations are shown in Fig. 9(a) only for samples of BC-BS for which we proved that the IRM ellipsoid can be used instead of the TRM one. The correction factor obtained through TRM anisotropy ellipsoid for all studied samples is given in Fig. 9(b). The correction factor is typically lower than 5\%. This is an unexpected result, especially for the brick's samples, which have a quite strong remanence anisotropy (Fig. 1).

According to Aitken et al. (1981) and Odah et al. (2001), the most important factor for the bias from the true palaeointensity is the angle between the NRM, carried by samples, and the imposed laboratory field. In our Thel- 


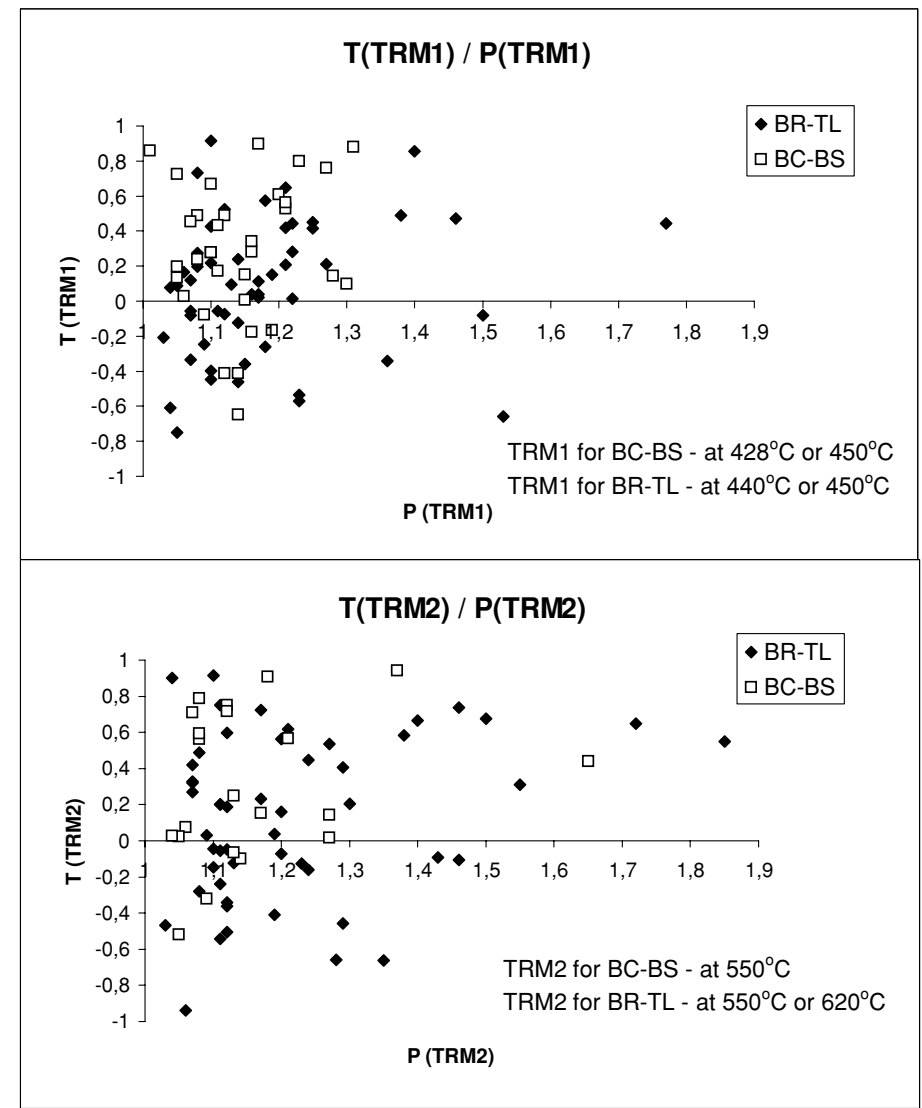

Fig. 4. Shape anisotropy parameter $T$ versus degree of anisotropy $P$ for baked clay/burnt soil (BC-BS) and for bricks/tiles (BR-TL) at the different temperatures shown in each graph.

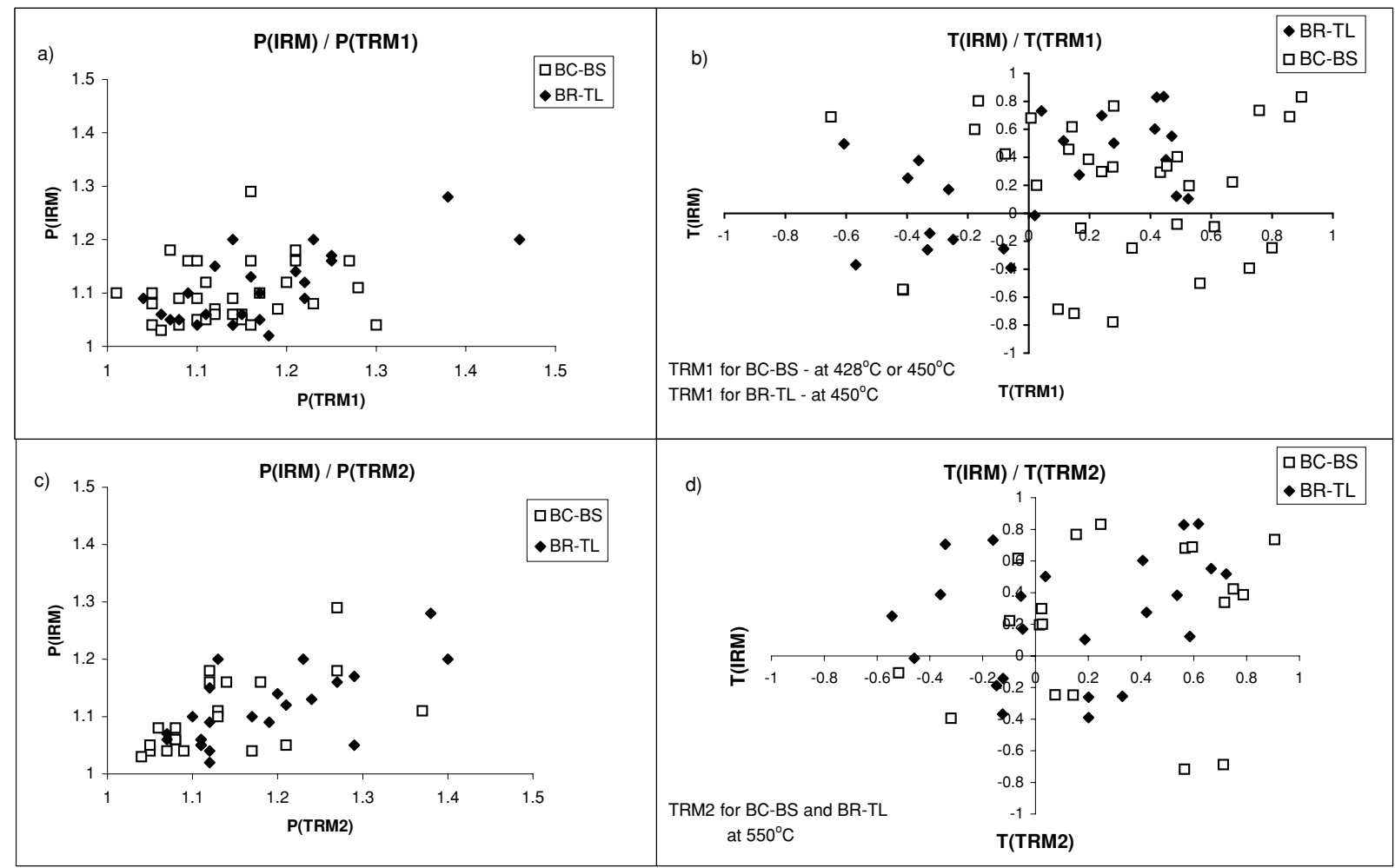

Fig. 5. Different relationships between degrees of anisotropy and shape parameters as obtained by IRM and TRM ellipsoids. 


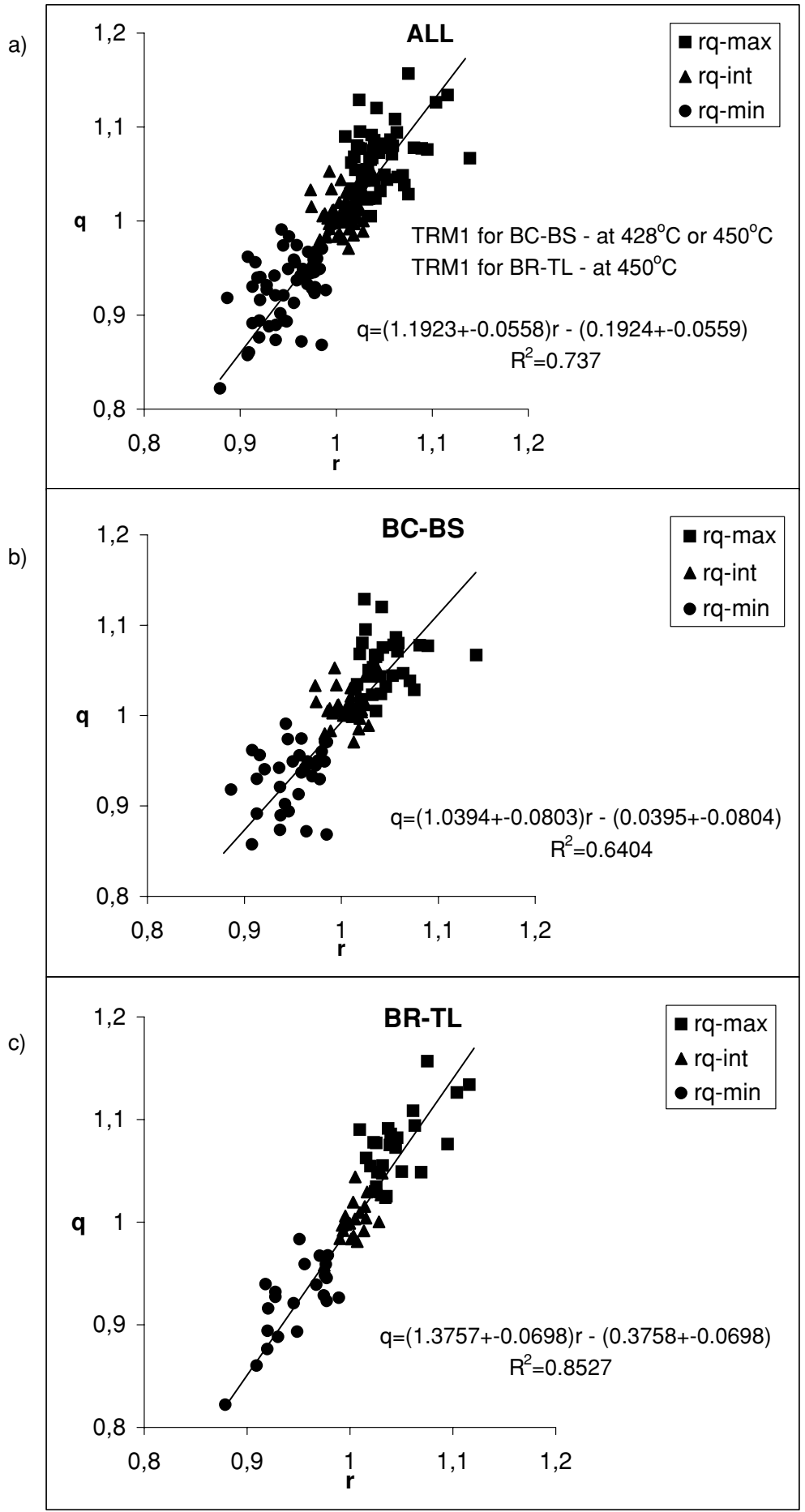

Fig. 6. Comparison of normalized principal axes' values of the IRM $\left(r_{x}=\operatorname{IRM}_{x x} /\left(\left(\operatorname{IRM}_{x x}+\operatorname{IRM}_{y y}+\mathrm{IRM}_{z z}\right) / 3\right)\right.$ etc. $)$ and $\mathrm{TRM}$ $\left(q_{x}=\mathrm{TRM}_{x x} /\left(\left(\mathrm{TRM}_{x x}+\mathrm{TRM}_{y y}+\mathrm{TRM}_{z z}\right) / 3\right)\right.$ etc.) ellipsoids at lower temperature (TRM1) level, as noted in: (a) all; (b) and (c) separately for different types of materials. The best fitted straight lines are given with their errors. The temperatures of TRM1 anisotropy evaluations for different experimental series are given as a legend in Fig. 6(a).

lier's experiment, the laboratory field is applied along the $Z$ axes of the samples (that is $I_{\mathrm{lab}}=90^{\circ}$ ). Knowing that the direction of $H_{\mathrm{lab}}$ is along the $Z$ axis, we define the angle $\left(90-I_{\mathrm{NRM}}\right)$ as the angular discrepancy between $H_{\text {lab }}$ and the carried NRM. We define the angle $\alpha$ as the angle between the direction of the maximum principal axis of the TRM anisotropy ellipsoid (given in Tables 1 and 2) and the NRM direction (given in Table 3). Finally, we define the correction, due to the magnetic anisotropy, as $|1-f|$.
Data reported in Fig. 10(a) indicate that the values of the correction factors do not depend on the angles between the NRM directions and the direction of maximum axes of the TRM anisotropy ellipsoids. This lack of systematic dependence on $\alpha$ is supported by the random directions of the $K_{\max }(\mathrm{TRM})$ for a given structure, reported recently by Gomez-Paccard et al. (2006) based on their study of baked clay materials from pottery kilns. The same is also evident from the 3D-plot in Fig. 10(b), where the parameter 


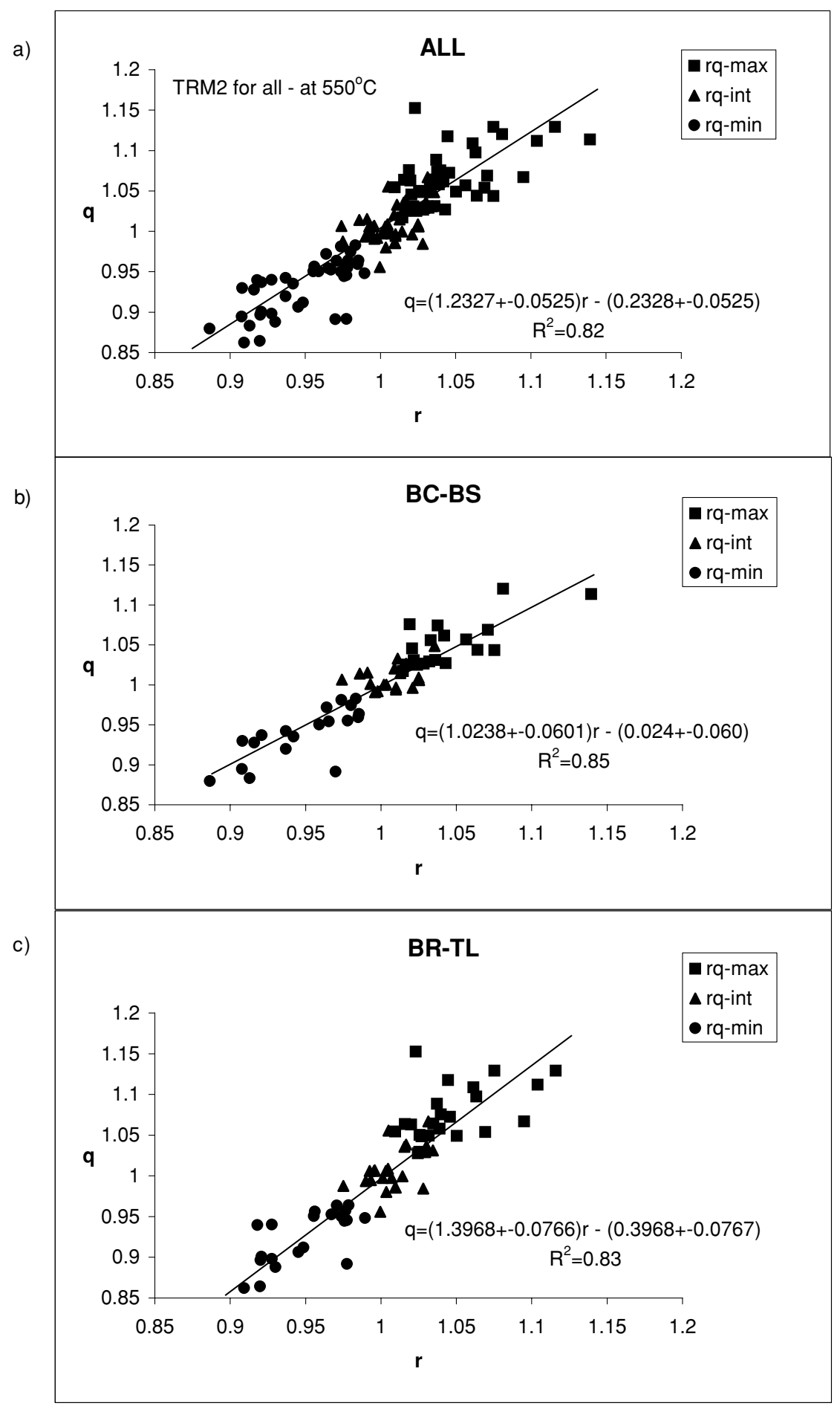

Fig. 7. As in Fig. 6 but for the higher temperature of $550^{\circ} \mathrm{C}$.

$P$ (degree of TRM anisotropy) is included as well. In the collection studied here, there are few cases of high $P$, which probably makes the upper part of the surface plot less well defined, but the trend is evident. In Fig. 11, a contourplot of the TRM anisotropy degree $P$, angular discrepancy between $H_{\mathrm{lab}}$, and the carried NRM $\left(90-I_{\mathrm{NRM}}\right)$, and the correction factor $|1-f|$ for all the samples is presented. The map is drawn using Kriging as an interpolation method. It appears that the correction factor $|1-f|$ increases with $P$ and $\left(90-I_{\mathrm{NRM}}\right)$.

In order to estimate the general relation between the two factors $\left(90-I_{\mathrm{NRM}}\right)$ and $P$ on the one hand and the obtained correction parameter $f$ (through $|1-f|$ ) on the other, the equation of the plane obtained by a polynomial regression of the 83 points was determined. The plane is defined as: $Z(X, Y)=A_{00}+A_{01} Y+A_{10} X$ with $A_{00}=-0.1239$; $A_{01}=0.1229 ; A_{10}=0.0100$, where $A_{01}$ is the coefficient for the degree of anisotropy and $A_{10}$ is the coefficient for the angular discrepancy between $H_{\text {lab }}$ and the carried NRM $\left(90-I_{\mathrm{NRM}}\right)$. In order to verify the relation, the isotropic case with $Y=1$ (i.e., $P=1.00)$ and $X=0$ (i.e., $(90-$ $\left.\left.I_{\mathrm{NRM}}\right)=0\right)$ is considered. Thus, $Z(0,1)=0.001$, which 
a)

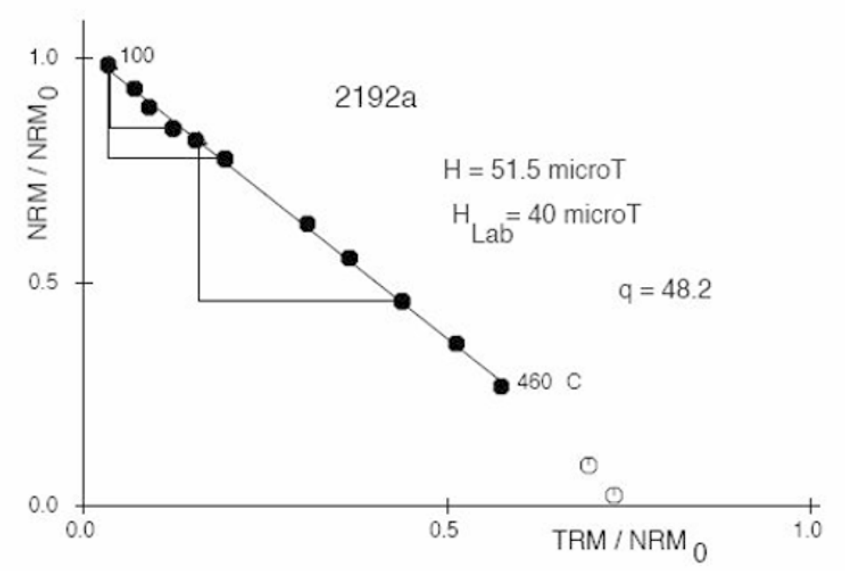

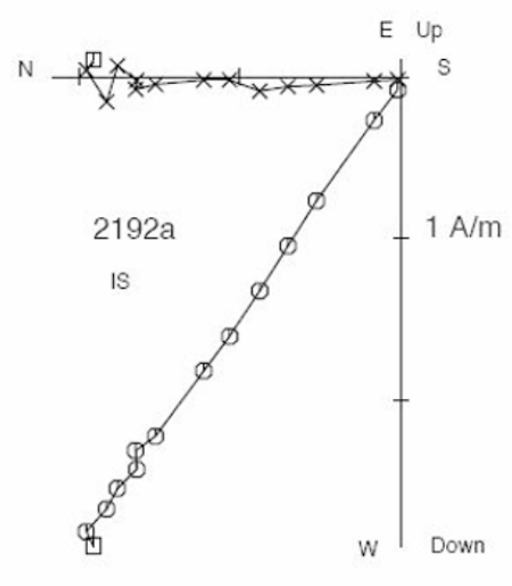

b)
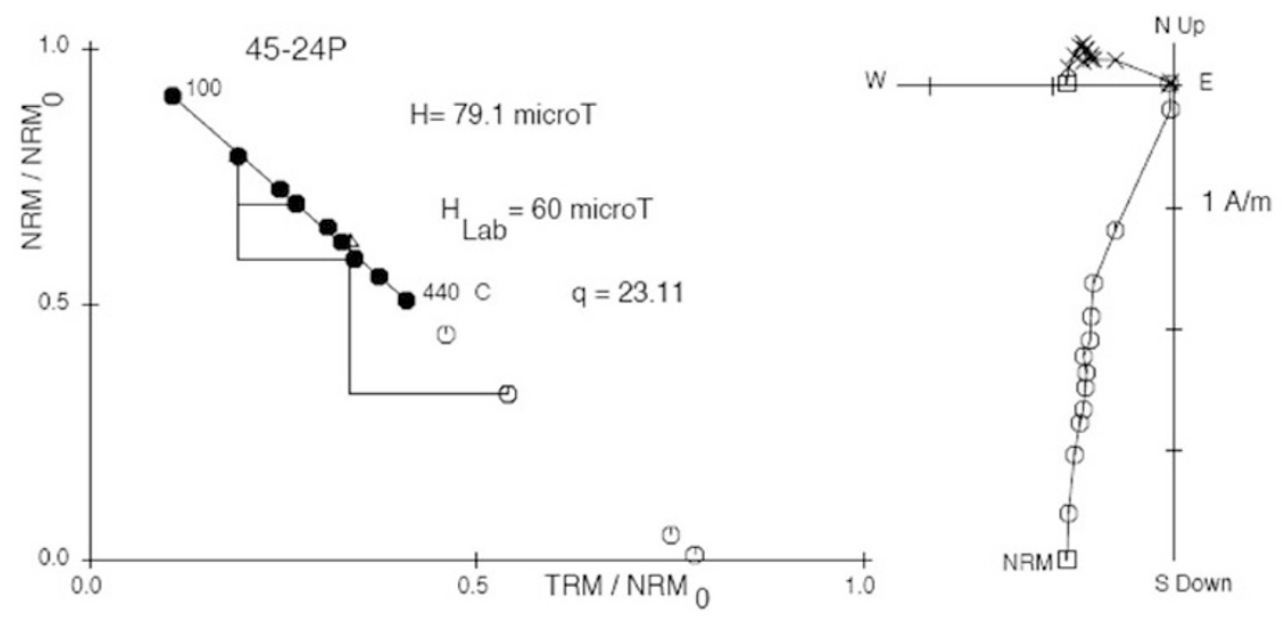

Fig. 8. Palaeointensity results of 2192a (BC-BS sample) and 45-24P (BR-TL sample) with the pTRM tests and Zijderveld diagrams. Points represented as solid circles are taken into the calculation of the regression line. The two-component remanence carried by the 45-24P tile in the kiln's construction is evident. The results shown are before anisotropy correction (Table 3). It is evident that the TRM1 evaluation is made in the temperature interval chosen for the palaeointensity determination.

clearly satisfies the physical meaning.

It is clear that the degree of anisotropy $P$ has the biggest impact on the correction factor, but the influence of the angular discrepancy between $H_{\text {lab }}$ and the carried NRM expressed by $\left(90-I_{\mathrm{NRM}}\right)$ cannot be neglected. Unfortunately, the programme used for the polynomial regression equation does not give us the standard errors of the calculated coefficients, and we cannot examine their statistical significance at this stage of study.

Figure 11 reveals that five points (marked with their $\alpha$ values) do not follow the general trend. A new calculation excluding these points leads to very similar coefficients, which are insignificantly different from the values calculated above. We conclude that these points have no important influence upon the trend observed between the parameters $P$ and $\left(90-I_{\mathrm{NRM}}\right)$, and the correcting factor.

However, it has been mentioned above that the upper part of the surface plot (Fig. 10(b)) is less well defined because of the low density of data with higher values of $P$. To estimate this ambiguity, we have recalculated the polynomial regression, excluding $P$ data higher than 1.3. The obtained new values of the regression coefficients using the remaining 75 points are as follows: $A_{00}=-0.1540$; $A_{01}=0.1494 ; A_{10}=0.0114$. Thus, the relation between them remains the same. The value of the regression coefficient $A_{01}$ (related to $P$ ) is again one order higher than that of the regression coefficient $A_{10}$ (related to the angular difference between the field directions of the NRM and those of the laboratory). This is a strong argument in support of the above-stated vision on the highest influence of $P$ values upon the anisotropy correction of the palaeointensity evaluations.

\section{Discussion}

Genevey and Gallet (2002) and Bowles et al. (2002) have recently reported that the TRM anisotropy effect on the 
Table 3. The effect of anisotropy correction on the obtained palaeointensity results (second, third and fourth columns). The age and the type of used material are specified in the last column. Temperat. Int. (deg) = temperature interval in degrees Celsius used to calculate the palaeointensity; Fa non-corr. = palaeointensity before correction; Fa corr. = palaeointensity after correction of anisotropy. When the average intensity per site is calculated, it is given with its dispersion. The change in the inter-sample dispersion in percentage is given below that corrected for the anisotropy site's palaeointensity. Remanence anisotropy parameters determined for the TRM and IRM tensors are shown in the fifth to eighth columns. $P(\mathrm{TRM})=$ degree of TRM anisotropy; $D(\mathrm{deg}), I(\mathrm{deg})(\mathrm{TRM})=$ declination (inclination) of the maximum axis of the TRM ellipsoid, in sample co-ordinates; $P($ IRM $)=$ degree of IRM anisotropy. The ninth and tenth columns show the correction factors $f$, determined through the TRM $(f$ $($ TRM $)$ ) and IRM ( $f(\operatorname{IRM}))$ tensors, respectively. The direction of the carried NRM vector is given in the eleventh and twelfth columns; $I$ (deg) and $D(\mathrm{deg})=$ inclination and declination of the NRM, respectively, in sample co-ordinates.

\begin{tabular}{|c|c|c|c|c|c|c|c|c|c|c|c|c|}
\hline \multirow{3}{*}{ No } & \multicolumn{3}{|c|}{ THELLIER EXPERIMENT } & \multicolumn{6}{|c|}{ REMANENCE ANISOTROPY } & \multicolumn{2}{|c|}{ NRM } & \multirow{3}{*}{$\begin{array}{c}\text { AGE } \\
\text { MATERIAL }\end{array}$} \\
\hline & Temperat. & Fa non-cor. & Fa cor. & $\mathrm{Kmax} / \mathrm{Kmin}$ & $\mathrm{K} \max (\mathrm{T}$ & RM) & $\mathrm{Kmax} / \mathrm{Kmin}$ & $\mathrm{f}$ & f & I & D & \\
\hline & int. (deg) & $($ microT) & (microT) & P(TRM1) & $\mathrm{D}(\mathrm{deg})$ & $\mathrm{I}(\mathrm{deg})$ & $\mathrm{P}(\mathrm{IRM})$ & (TRM1) & (IRM) & $(\mathrm{deg})$ & (deg) & \\
\hline $2192 \mathrm{a}$ & $100-460$ & 51.48 & 51.64 & 1.11 & 31 & 60 & 1.12 & 1.00 & 1.06 & 55 & 359 & $13 \mathrm{c} \cdot \mathrm{AD}$ \\
\hline $2192 b$ & $180-430$ & 57.25 & 57.97 & 1.20 & 351 & 75 & 1.12 & 1.01 & 0.98 & 60 & 7 & baked clay \\
\hline $2168 \mathrm{a}$ & $100-500$ & 58.51 & 61.37 & 1.17 & 108 & 32 & 1.10 & 1.05 & 1.05 & 58 & 18 & $11 \mathrm{c} . \mathrm{AD}$ \\
\hline $2215 b$ & $100-500$ & 51.74 & 51.64 & 1.01 & 218 & 0 & 1.10 & 1.00 & 1.03 & 61 & 13 & $\begin{array}{l}11-12 \text { c. AD } \\
\text { baked clay }\end{array}$ \\
\hline $2221 b$ & $220-500$ & 58.83 & 59.27 & 1.12 & 237 & 44 & 1.06 & 1.01 & 1.00 & 68 & 340 & 9 c. $\mathrm{AD}$ \\
\hline $2223 b$ & $190-550$ & 66.2 & 67.28 & 1.16 & 115 & 25 & 1.04 & 1.02 & 1.00 & 64 & 346 & baked clay \\
\hline $2073 a$ & $100-550$ & 69.43 & 66.35 & 1.30 & 261 & 43 & 1.04 & 0.96 & 1.00 & 61 & 332 & $\begin{array}{l}\text { ca } 4 \text { c. BC } \\
\text { burnt soil }\end{array}$ \\
\hline $2085 v$ & $100-500$ & 86.65 & 93.33 & 1.28 & 79 & 51 & 1.11 & 1.08 & 1.05 & 58 & 340 & ca 4 c. BC \\
\hline $2085 \mathrm{~g}$ & $100-460$ & 89.32 & 96.61 & 1.16 & 112 & 64 & 1.16 & 1.08 & 1.06 & 56 & 340 & burnt soil \\
\hline $2241 \mathrm{a}$ & $100-500$ & 69.33 & 67.56 & 1.09 & 173 & 1 & 1.16 & 0.97 & 0.96 & 56 & 353 & ca 4 c. BC \\
\hline $2242 a$ & $235-428$ & 62.68 & 65.01 & 1.21 & 54 & 22 & 1.16 & 1.04 & 0.98 & 50 & $260 ?$ & burnt soil \\
\hline $2253 \mathrm{a}$ & $100-550$ & 78.45 & 75.32 & 1.10 & 131 & 3 & 1.09 & 0.96 & 0.95 & 46 & 345 & ca 4 c. BC \\
\hline $2264 v$ & paleoint. & result & discarded & 1.21 & 241 & 24 & 1.18 & 1.03 & 0.96 & 61 & 342 & burnt soil \\
\hline I $9 v$ & $100-398$ & 44.59 & 45.84 & 1.10 & 238 & 46 & 1.05 & 1.03 & 1.02 & 59 & 342 & ca 4 c. BC? \\
\hline I $11 \mathrm{~b}$ & $100-398$ & 38.85 & 39.46 & 1.15 & 260 & 44 & 1.06 & 1.02 & 1.02 & 66 & 5 & baked clay \\
\hline CA54a & $100-460$ & 44.53 & 39.88 & 1.31 & 156 & 2 & 1.11 & 0.90 & 1.02 & 45 & 342 & $\begin{array}{c}\text { Bronze } \\
\text { baked clay }\end{array}$ \\
\hline D $174 \mathrm{~g}$ & $100-398$ & 47.59 & 47.68 & 1.15 & 152 & 19 & 1.05 & 1.00 & 1.03 & 57 & 355 & Bronze \\
\hline D192v & $180-430$ & 47.2 & 46.02 & 1.08 & 65 & 14 & 1.09 & 0.98 & 0.97 & 54 & 354 & baked clay \\
\hline GV33b & $100-430$ & 61.1 & 60.88 & 1.05 & 179 & 0 & 1.10 & 1.00 & 1.02 & 62 & 350 & Bronze \\
\hline GV45v & $100-460$ & 60.02 & 61.86 & 1.23 & 124 & 40 & 1.08 & 1.03 & 1.02 & 57 & 352 & baked clay \\
\hline $2145 b$ & $100-550$ & 63.36 & 68.59 & 1.19 & 126 & 44 & 1.07 & 1.08 & 0.99 & 65 & 309 & $\begin{array}{c}\text { Iron } \\
\text { burnt soil }\end{array}$ \\
\hline $86 v$ & $230-540$ & 65.72 & 65.71 & 1.06 & 101 & 4 & 1.03 & 1.00 & 0.99 & 62 & 3 & $\begin{array}{l}\text { end } 6 \mathrm{c} . \mathrm{AD} \\
\text { baked clay }\end{array}$ \\
\hline DG37d & $100-460$ & 63.41 & 62.43 & 1.14 & 140 & 19 & 1.09 & 0.98 & 1.03 & 65 & 183 & ca 4c.BC? \\
\hline DG45d & paleoint. & result & discarded & 1.12 & 110 & 28 & 1.07 & 0.99 & 1.01 & 72 & 356 & baked clay \\
\hline DG23e & paleoint. & result & discarded & 1.05 & 212 & 13 & 1.08 & 1.00 & 1.00 & 70 & 329 & \\
\hline $94 b$ & $230-540$ & 72.3 & 72.23 & 1.14 & 175 & 1 & 1.06 & 1.00 & 1.01 & 78 & 330 & $\begin{array}{c}10 \mathrm{c} . \mathrm{AD} \\
\text { baked clay }\end{array}$ \\
\hline $101 \mathrm{v}$ & $190-540$ & 79.34 & 79.34 & 1.08 & 57 & 29 & 1.04 & 1.00 & 1.00 & 74 & 20 & $\begin{array}{c}10 \mathrm{c} . \mathrm{AD} \\
\text { baked clay }\end{array}$ \\
\hline
\end{tabular}


Table 3. (continued).

\begin{tabular}{|c|c|c|c|c|c|c|c|c|c|c|c|c|}
\hline \multirow[b]{2}{*}{ No } & \multicolumn{3}{|c|}{ THELLIER EXPERIMENT } & \multicolumn{6}{|c|}{ REMANENCE ANISOTROPY } & \multicolumn{2}{|c|}{ NRM } & \multirow{2}{*}{$\begin{array}{c}\text { AGE } \\
\text { MATERIAL }\end{array}$} \\
\hline & $\begin{array}{l}\text { Temperat. } \\
\text { int. (deg) }\end{array}$ & $\begin{array}{c}\text { Fa non-cor. } \\
\text { (microT) }\end{array}$ & $\begin{array}{c}\text { Fa cor. } \\
\text { (microT) }\end{array}$ & $\begin{array}{c}\mathrm{Kmax} / \mathrm{Kmin} \\
\mathrm{P}(\mathrm{TRM} 1)\end{array}$ & $\begin{array}{l}\mathrm{K} \max (\mathrm{T} \\
\mathrm{D}(\mathrm{deg})\end{array}$ & $\begin{array}{l}\text { RM) } \\
\text { I (deg) }\end{array}$ & $\begin{array}{c}\mathrm{Kmax} / \mathrm{Kmin} \\
\mathrm{P}(\mathrm{IRM})\end{array}$ & $\begin{array}{c}\mathrm{f} \\
(\mathrm{TRM} 1)\end{array}$ & $\begin{array}{c}\mathrm{f} \\
(\text { IRM) } \\
\end{array}$ & $\begin{array}{l}\text { I } \\
\text { (deg) }\end{array}$ & $\begin{array}{c}\text { D } \\
(\mathrm{deg}) \\
\end{array}$ & \\
\hline $641 \mathrm{a}$ & $190-550$ & 83.66 & 83.94 & 1.11 & 81 & 39 & 1.05 & 1.00 & 1.00 & 72 & 338 & $641-$ \\
\hline $642 b$ & $190-540$ & 73.62 & 72.67 & 1.07 & 44 & 8 & 1.18 & 0.99 & 0.98 & 71 & 342 & $647 \mathrm{AD}$ \\
\hline $1576 \mathrm{a}$ & $190-540$ & 83.01 & 82.3 & 1.16 & 53 & 45 & 1.29 & 0.99 & 1.02 & 67 & 143 & burnt soil \\
\hline & average & $80.1+-5.62$ & $\begin{array}{c}79.64+-6.09 \\
-\mathbf{8} \%\end{array}$ & & & & & & & & & \\
\hline $1788 \mathrm{a}$ & $190-450$ & 68.5 & 65.89 & 1.27 & 100 & 1 & 1.16 & 0.96 & 0.97 & 67 & 17 & $\begin{array}{c}10 \mathrm{c} . \mathrm{AD} \\
\text { baked clay }\end{array}$ \\
\hline $1509 \mathrm{a}$ & paleoint. & result & discarded & 1.05 & 267 & 9 & 1.08 & 1.03 & 1.01 & -40 & 357 & $\begin{array}{l}7 \mathrm{c} . \mathrm{AD} \\
\text { burnt soil }\end{array}$ \\
\hline $69 \mathrm{~b}$ & $190-420$ & 56.24 & 56.51 & 1.08 & 353 & 46 & 1.05 & 1.01 & 1.00 & 57 & 59 & $5-6 \mathrm{c} . \mathrm{AD}$ \\
\hline $70 \mathrm{~b}$ & $190-450$ & 61.26 & 59.08 & 1.09 & 198 & 10 & 1.10 & 0.96 & 0.97 & -52 & 39 & bricks \\
\hline $268 \mathrm{a}$ & $190-550$ & 65.65 & 62.65 & 1.25 & 170 & 1 & 1.16 & 0.95 & 0.97 & -24 & 175 & 5-6 c. AD \\
\hline $269 \mathrm{a}$ & $190-450$ & 57.98 & 60.98 & 1.38 & 171 & 50 & 1.28 & 1.05 & 1.13 & -5 & 160 & bricks \\
\hline $270 \mathrm{a}$ & $\begin{array}{l}230-540 \\
\text { average }\end{array}$ & $\begin{array}{c}55.93 \\
59.85+-5.1\end{array}$ & $\begin{array}{c}58.65 \\
60.76+-2.01 \\
\mathbf{6 1 \%}\end{array}$ & 1.22 & 188 & 7 & 1.12 & 1.05 & 1.04 & 67 & 300 & \\
\hline $302 v$ & $190-450$ & 67.82 & 65.59 & 1.25 & 6 & 9 & 1.17 & 0.97 & 0.97 & 58 & 340 & $6 \mathrm{c} . \mathrm{AD}$ \\
\hline $305 \mathrm{a}$ & $230-450$ & 58.36 & 59.53 & 1.46 & 353 & 3 & 1.20 & 1.02 & 1.01 & 65 & 315 & bricks \\
\hline $376 \mathrm{~b}$ & $190-450$ & 58.17 & 57.21 & 1.21 & 170 & 2 & 1.14 & 0.98 & 1.02 & 24 & 107 & $6 \mathrm{c} . \mathrm{AD}$ \\
\hline $379 \mathrm{a}$ & $190-420$ & 59.78 & 60.33 & 1.06 & 285 & 60 & 1.06 & 1.01 & 1.01 & 12 & 340 & bricks \\
\hline $551 \mathrm{~b}$ & $190-450$ & 63.29 & 62.62 & 1.07 & 39 & 2 & 1.07 & 0.99 & 0.99 & 60 & 359 & $6 \mathrm{c} . \mathrm{AD}$ \\
\hline $552 \mathrm{a}$ & $100-400$ & 69.84 & 69.97 & 1.08 & 40 & 16 & & 1.00 & & & & bricks in \\
\hline $1609 \mathrm{a}$ & paleoint. & result & discarded & 1.14 & 112 & 40 & 1.21 & 1.03 & 1.1 & 34 & 26 & $6 \mathrm{c} \cdot \mathrm{AD}$ \\
\hline $1596 \mathrm{v}$ & $100-510$ & 56.67 & 62.62 & 1.77 & 193 & 65 & & 1.11 & & -15 & 5.4 & bricks \\
\hline B175b & $100-440$ & 59.06 & 60 & 1.03 & 207 & 50 & & 1.02 & & 14 & 330 & $5 \mathrm{c} . \mathrm{AD}$ \\
\hline B178a & $200-440$ & 55.5 & 55.33 & 1.13 & 193 & 29 & & 1.00 & & -53 & 280 & bricks \\
\hline $\mathrm{B} 182 \mathrm{~b}$ & $\begin{array}{l}100-510 \\
\text { average }\end{array}$ & $\begin{array}{c}61.8 \\
58.79+-3.16\end{array}$ & $\begin{array}{c}66.6 \\
60.64+-5.66 \\
-\mathbf{3 6} \%\end{array}$ & 1.12 & 184 & 62 & & 1.08 & & -22 & 210 & \\
\hline B207b & $150-360$ & 73 & 69.1 & 1.07 & 153 & 20 & & 0.95 & & -3 & 330 & $6 \mathrm{c} . \mathrm{AD}$ \\
\hline B210a & $100-360$ & 57.42 & 58.65 & 1.07 & 248 & 58 & & 1.02 & & 60 & 120 & bricks \\
\hline $\mathrm{B} 211 \mathrm{a}$ & $\begin{array}{l}100-480 \\
\text { average }\end{array}$ & $\begin{array}{c}62.82 \\
64.41+-7.91\end{array}$ & $\begin{array}{c}62.3 \\
63.35+-5.3 \\
\mathbf{3 3} \%\end{array}$ & 1.04 & 329 & 3 & & 0.99 & & 60 & 298 & \\
\hline $36-3$ & paleoint. & result & discarded & 1.11 & 274 & 68 & 1.06 & 1.00 & 1.01 & 73 & 5 & $7-8 \mathrm{c} . \mathrm{AD}$ \\
\hline $36-8$ & $190-550$ & 75.53 & 73.1 & 1.12 & 294 & 19 & 1.15 & 0.97 & 0.99 & 66 & 17 & tiles in \\
\hline $36-13$ & $190-550$ & 79.38 & 74.79 & 1.17 & 14 & 29 & 1.10 & 0.94 & 0.99 & 64 & 2 & kiln's \\
\hline $36-13 a$ & $100-480$ & 83.82 & 78.91 & 1.17 & 210 & 24 & & 0.94 & & -62 & 17 & construction \\
\hline $36-16$ & $190-550$ & 75.64 & 74.91 & 1.17 & 222 & 9 & 1.05 & 0.99 & 0.99 & 68 & 10 & \\
\hline $36-17$ & $190-420$ & 75.13 & 75.18 & 1.04 & 210 & 11 & 1.09 & 1.00 & 1.01 & 66 & 10 & \\
\hline $36-19$ & $190-550$ & 73.97 & 73.57 & 1.10 & 162 & 9 & 1.05 & 0.99 & 1.00 & 70 & 13 & \\
\hline $36-19 \mathrm{a}$ & $\begin{array}{l}240-510 \\
\text { average }\end{array}$ & $\begin{array}{c}81.95 \\
77.92+-3.82\end{array}$ & $\begin{array}{c}81.82 \\
76.04+-3.16 \\
17 \%\end{array}$ & 1.10 & 322 & 45 & & 1.00 & & -73 & 196 & \\
\hline
\end{tabular}

palaeointensity determinations from pottery lead to correction factors of up to $30 \%$. This has been experimentally shown by Odah et al. (2001) for pottery in which the easy plane seems to play the major role. Our results indicate that the effect of the anisotropy upon palaeointensity de- terminations is lower on BR-TL and lower still on BC-BS than on pottery, as mentioned in previous comparative studies on the basis of less numerous collections (Jordanova et al., 1995; Kovacheva et al., 1998). Furthermore, in an archaeomagnetic study of Danish materials from bricks, a few 
Table 3. (continued).

\begin{tabular}{|c|c|c|c|c|c|c|c|c|c|c|c|c|}
\hline No & $\begin{array}{l}\text { THELL } \\
\text { Temperat. } \\
\text { int. (deg) }\end{array}$ & $\begin{array}{l}\text { R EXPERIMEN } \\
\text { Fa non-cor. } \\
\text { (microT) }\end{array}$ & $\begin{array}{l}\text { Fa cor. } \\
\text { (microT) }\end{array}$ & $\begin{array}{c}\mathrm{Kmax} / \mathrm{Kmin} \\
\mathrm{P}(\mathrm{TRM} 1)\end{array}$ & $\begin{array}{l}\text { REMAl } \\
\text { Kmax (T } \\
\text { D (deg) }\end{array}$ & $\begin{array}{l}\text { JENCE } \\
\text { RM) } \\
\text { I (deg) }\end{array}$ & $\begin{array}{l}\text { ANISOTROP } \\
\text { Kmax/Kmin } \\
\text { P(IRM) }\end{array}$ & $\begin{array}{c}\text { Y } \\
\text { f } \\
\text { (TRMI) }\end{array}$ & $\begin{array}{c}\mathrm{f} \\
(\mathrm{IRM})\end{array}$ & $\begin{array}{c}\mathrm{N} \\
\mathrm{I} \\
(\mathrm{deg})\end{array}$ & $\begin{array}{c}\mathrm{D} \\
\mathrm{D} \\
(\mathrm{deg})\end{array}$ & $\begin{array}{c}\text { AGE } \\
\text { MATERIAL }\end{array}$ \\
\hline 41-1 & $190-450$ & 92 & 90.13 & 1.23 & 48 & 10 & 1.20 & 0.98 & 0.93 & 63 & 13 & 6-7 c. AD \\
\hline $41-4$ & $190-490$ & $129.21^{*}$ & $126.63^{*}$ & 1.16 & 52 & 12 & 1.13 & 0.98 & 0.99 & 65 & 14 & tiles in \\
\hline $41-6$ & paleoint. & result & discarded & 1.14 & 53 & 2 & 1.04 & 1.01 & 0.99 & 68 & 4 & kiln's \\
\hline $41-6 a$ & $240-480$ & 100.43 & 99 & 1.08 & 86 & 11 & & 0.99 & & -67 & 41 & construction \\
\hline $41-8$ & $230-420$ & 99.35 & 100.35 & 1.18 & 225 & 16 & 1.02 & 1.01 & 1.00 & 73 & 15 & \\
\hline $41-8 \mathrm{a}$ & $20-480$ & 108.88 & 109.97 & 1.18 & 48 & 3 & & 1.01 & & -70 & 0 & \\
\hline $41-9$ & $190-550$ & 99.98 & 97.1 & 1.22 & 157 & 0 & 1.09 & 0.97 & 0.98 & 68 & 9 & \\
\hline $41-19$ & paleoint. & result & discarded & 1.15 & 25 & 30 & 1.06 & 0.97 & 0.99 & 66 & 4 & \\
\hline $41-19 \mathrm{a}$ & $300-480$ & $79.57^{*}$ & $79 *$ & 1.07 & 215 & 8 & & 0.99 & & -66 & 140 & \\
\hline & average & $100.04+-5.99$ & $\begin{array}{c}99.31+-7.14 \\
-16 \%\end{array}$ & & & & & & & & & \\
\hline $45-8 \mathrm{P}$ & $20-510$ & 78.95 & 81.41 & 1.10 & 87 & 12 & & 1.03 & & 55 & 194 & 6-7 c. AD \\
\hline $45-12 \mathrm{P}$ & $100-300$ & 78.18 & 79.33 & 1.14 & 40 & 2 & & 1.01 & & 85 & 220 & tiles in \\
\hline $45-15 \mathrm{P}$ & paleoint. & result & discarded & 1.19 & 33 & 4 & & & & & & kiln's \\
\hline $45-16 \mathrm{P}$ & paleoint. & result & discarded & 1.23 & 217 & 42 & & & & & & construction \\
\hline 45-17P & $100-510$ & 82.95 & 81.52 & 1.22 & 126 & 2 & & 0.98 & & 67 & 208 & \\
\hline $45-18 \mathrm{P}$ & $100-510$ & 84.55 & 84.38 & 1.21 & 131 & 40 & & 1.00 & & 69 & 197 & \\
\hline 45-19P & $150-510$ & $69.98^{*}$ & $69.36^{*}$ & 1.05 & 112 & 7 & & 0.99 & & 73 & 203 & \\
\hline $45-20 \mathrm{P}$ & $100-560$ & 82.38 & 82.02 & 1.05 & 158 & 9 & & 1.00 & & 71 & 196 & \\
\hline $45-21 \mathrm{P}$ & $150-360$ & 73.65 & 77 & 1.27 & 115 & 21 & & 1.04 & & 70 & 178 & \\
\hline $45-22 \mathrm{P}$ & $100-440$ & 83.63 & 84.07 & 1.17 & 300 & 11 & & 1.01 & & 75 & 220 & \\
\hline $45-23 \mathrm{P}$ & $100-510$ & 86.42 & 86.44 & 1.10 & 26 & 21 & & 1.00 & & 69 & 220 & \\
\hline $45-24 \mathrm{P}$ & $100-440$ & 79.06 & 78.75 & 1.10 & 136 & 12 & & 1.00 & & 72 & 286 & \\
\hline $45-25 \mathrm{P}$ & $100-560$ & 81.11 & 80.65 & 1.36 & 43 & 5 & & 0.99 & & 74 & 206 & \\
\hline $45-26 \mathrm{P}$ & $\begin{array}{l}100-560 \\
\text { average }\end{array}$ & $\begin{array}{c}79 \\
81.07+-3.58\end{array}$ & $\begin{array}{c}80.41 \\
81.45+-2.72 \\
24 \%\end{array}$ & 1.21 & 139 & 3 & & 1.02 & & 73 & 197 & \\
\hline 34-1P & $100-560$ & 74.75 & 73.92 & 1.18 & 298 & 14 & & 0.99 & & 55 & 90 & $4-5 \mathrm{c} . \mathrm{AD}$ \\
\hline $34-12 P$ & $100-400$ & 72.85 & 70.82 & 1.40 & 21 & 2 & & 0.97 & & -47 & 330 & bricks in \\
\hline $34-15 \mathrm{P}$ & $100-620$ & 80.41 & 72.86 & 1.53 & 321 & 11 & & 0.91 & & 49 & 348 & kiln's \\
\hline $34-16 \mathrm{P}$ & $\begin{array}{l}100-440 \\
\text { average }\end{array}$ & $\begin{array}{c}69.55 \\
74.39+-4.55\end{array}$ & $\begin{array}{c}65.35 \\
70.74+-3.82 \\
16 \%\end{array}$ & 1.50 & 242 & 0 & & 0.94 & & 56 & 358 & construction \\
\hline
\end{tabular}

ceramics, and burned clay from furnaces, Gram-Jensen et al. (2000) proved the negligible influence of the remanence anisotropy estimated by TRM anisotropy tensor, as has been done in the present study. In a more recent paper, Genevey et al. (2003) estimated the TRM anisotropy correction on the palaeointensity from both pottery and bricks from Syria. The obtained correction factor for bricks was found to cluster between 0.98 and 1.02 for $70 \%$ of the specimens, which is in excellent agreement with the results shown in this study. However, Leino and Pesonen (1994) reported stronger remanence anisotropy in bricks than in potsherds, but their conclusion was drawn on a very small number of experiments.

In a palaeointensity study on older potsherds from Ontario, Yu and Dunlop (2000) concluded that there is no correlation between the degree of remanence anisotropy and the estimated palaeointensity value. However, in our study, we were able to show that the values of the correcting factor of palaeointensity determinations, even if is low, depend on the degree of remanent anisotropy and also on the angle between the NRM direction and the laboratory field direction. In the recently developed new three-axis magnetome- ter (Le Goff and Gallet, 2004), the correction for anisotropy effect is automatically done by keeping the angle between the NRM and laboratory field direction less than $4^{\circ}$. This recent technological development will be a valuable tool and add precision to the obtained palaeointensity results from the most anisotropic objects such as pottery fragments (Rogers et al., 1979; Aitken et al., 1981; Jordanova et al., 1995). Our study has shown that when a difference between the applied laboratory field and that carried by the sample NRM exists, which is always the case in practice, the degree of anisotropy also plays an important role. As it can be seen in Table 3 , the angle between the $I_{\mathrm{NRM}}$ and the laboratory field is not very large in most cases. Thus, despite a high degree of remanence anisotropy $\left(K_{\max } / K_{\min }\right)$, the obtained correction factor $f_{\text {TRM }}$ is not high (Table 3: samples GV45v; 94b; 1576a; 302a; 305a; 41-1; 41-9; 45$17 \mathrm{P}, 18 \mathrm{P}, 21 \mathrm{P}, 25 \mathrm{P})$. However, large correction factors $f_{\mathrm{TRM}}$ are observed on a few samples which have a strong magnetic anisotropy and which carry a NRM in a direction that is far from the laboratory field direction (Table 3: samples CA54a; 269a; 1596v; B182b; 34-15P). The opposite is true in cases when the NRM direction is entirely different from 
a)

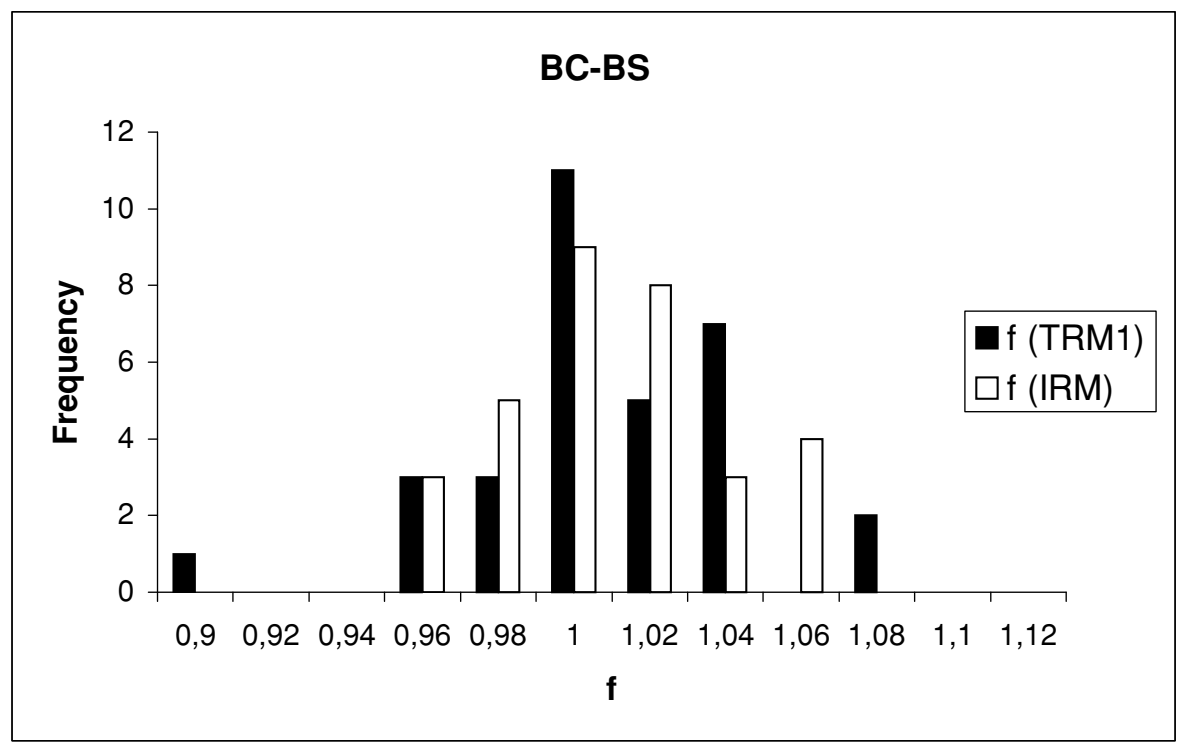

b)

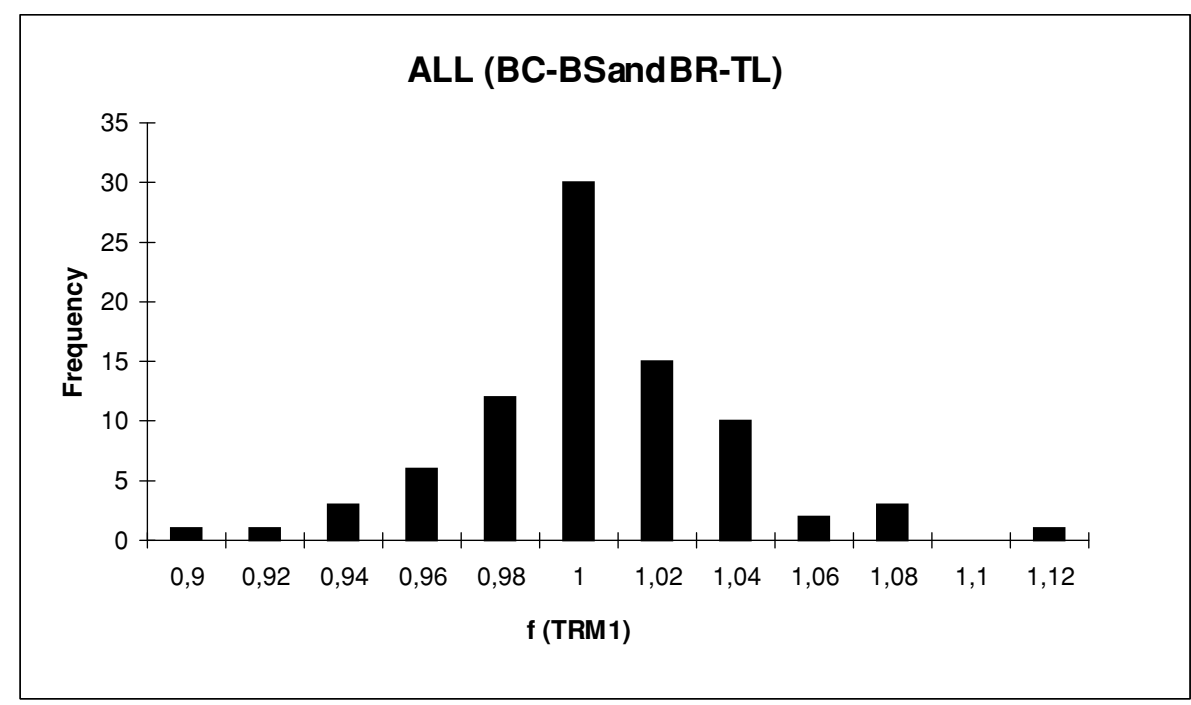

Fig. 9. (a) Histogram of frequency distribution of the obtained values of correction factor $f$ determined through the anisotropy estimation of TRM and IRM tensors for BC-BS; (b) frequency distribution of the obtained values of correction factor $f$ determined through TRM tensor at the intermediate (first) temperature for all studied samples.

the laboratory field but the degree of remanence anisotropy remains low; the correction factor is also not significant (Table 3: samples 1509a; 379a; B175b).

In Table 3 , the average values of palaeointensity per sites, before and after the correction of anisotropy, are also reported when more than two samples were studied per site. In five of the seven cases of sites presented by BR-TL, the standard deviations around the means decrease after the corrections, although the average values themselves are not affected substantially. The change in the inter-sample dispersion after the anisotropy correction is given in percentage in the fourth column of Table 3, where the minus sign means a worsening of the dispersion. Looking closely at these figures, we can make a number of remarks. The BS samples of the first site have NRM directions (11th column) close to the direction of the laboratory field, and the anisotropy effect should be smaller, which is the case $(-8 \%)$. The next site represented by bricks with a considerable degree of remanence anisotropy (fifth column) and two samples hav- ing NRM directions far away of the laboratory field direction shows the largest amelioration of the dispersion (61\%). The two last sites presented in Table 3, having a substantial degree of TRM anisotropy, have different correction factors (ninth column), depending on the mutual orientation of NRM and laboratory field. In these two cases, the dispersion is also improved (24 and 16\%), when sample 34$15 \mathrm{P}$, with the highest $P$ (TRM) and NRM's largest deviation from the laboratory field direction for these two sites, has the strongest correction factor. Thus, in order to obtain the best palaeointensity data on bricks and tiles, either a correction for anisotropy is needed or the laboratory field must be applied along the NRM direction. As far as baked clay plasters of ovens and kilns are concerned, we can trust the multiple published palaeointensity data without anisotropy correction, taking into account the results reported here. In fact, our results show that the anisotropy correction is small/insignificant for BC-BS materials and that these materials can be considered as isotropic. There re- 
a)

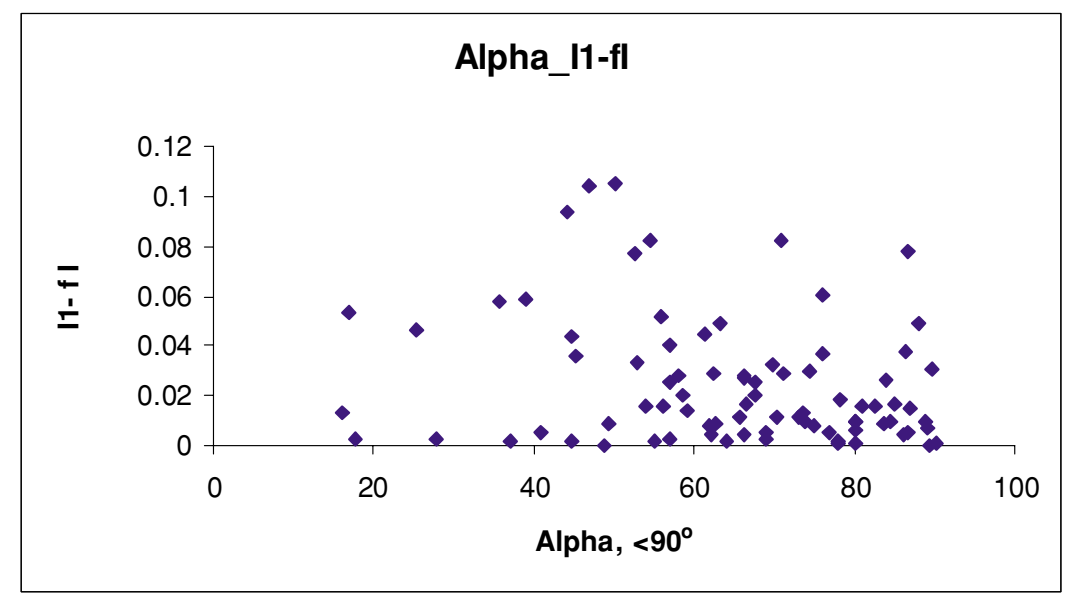

b)

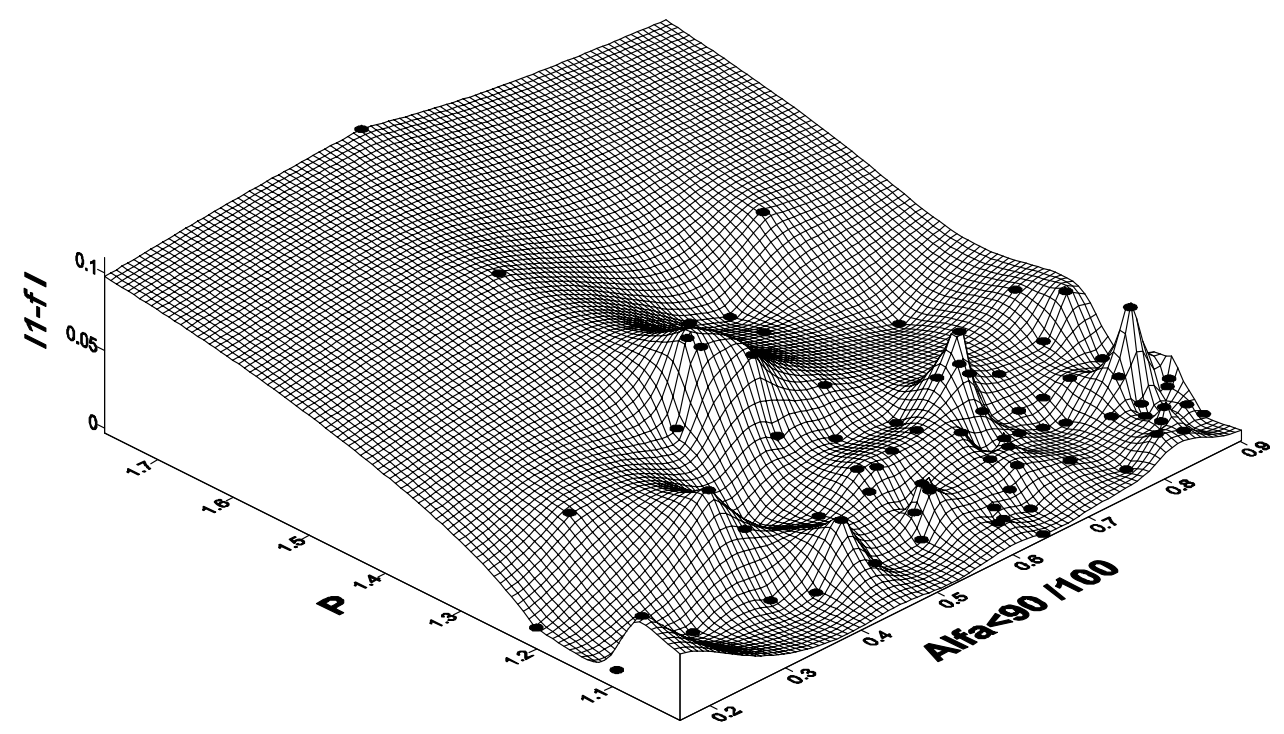

Fig. 10. (a) The anisotropy correction on the palaeointensity values as a function of the angle between NRM and the maximum axis of the TRM anisotropy ellipsoid ( $\alpha$ ); (b) 3-D plot representing the mutual influence of the degree of TRM anisotropy $P$ and $\alpha$ on the correction of palaeointensity value $|1-f|$. The values $\alpha$ on the $X$ axis have been divided by 100 for better visualization.

sults, therefore, can be used in further compilation of worldwide palaeointensity data with an equal weight as that corrected for anisotropy results from, for example, potteries.

To avoid the unwanted multiple heating for TRM ellipsoid estimation, our results show that the laboratoryinduced IRM remanence could be used instead when materials are baked clay/burnt soils. Only for this kind of material we have obtained a confirmation for the similarity of the anisotropy tensors shape of IRM and TRM remanence (Figs. 6(b) and 7(b)). In other words, replacing the TRM ellipsoid with the IRM one in the case of the BR-TL samples as a result of studied collection is not straightforward (Figs. 6(c) and 7(c)) despite the observed similarity of the principal axes directions in most of the cases. Hus et al.
(2002), comparing the TRM and anhysteretic anisotropy ellipsoids (ARM) for bricks, come to the same conclusion, thereby confirming the similarity of the principal axes directions, but changes in the shape. They found an equality in ellipsoid shapes only between that of TRM and that of partial anhysteretic remanence (PARM).

\section{Conclusions}

1. The degree of remanence anisotropy of baked clay/burnt soil is significantly lower than that in bricks and tiles, which have shape anisotropy.

2. The comparison of remanence anisotropy at two temperature levels reveals that the tensor determination through measurements on three perpendicular direc- 


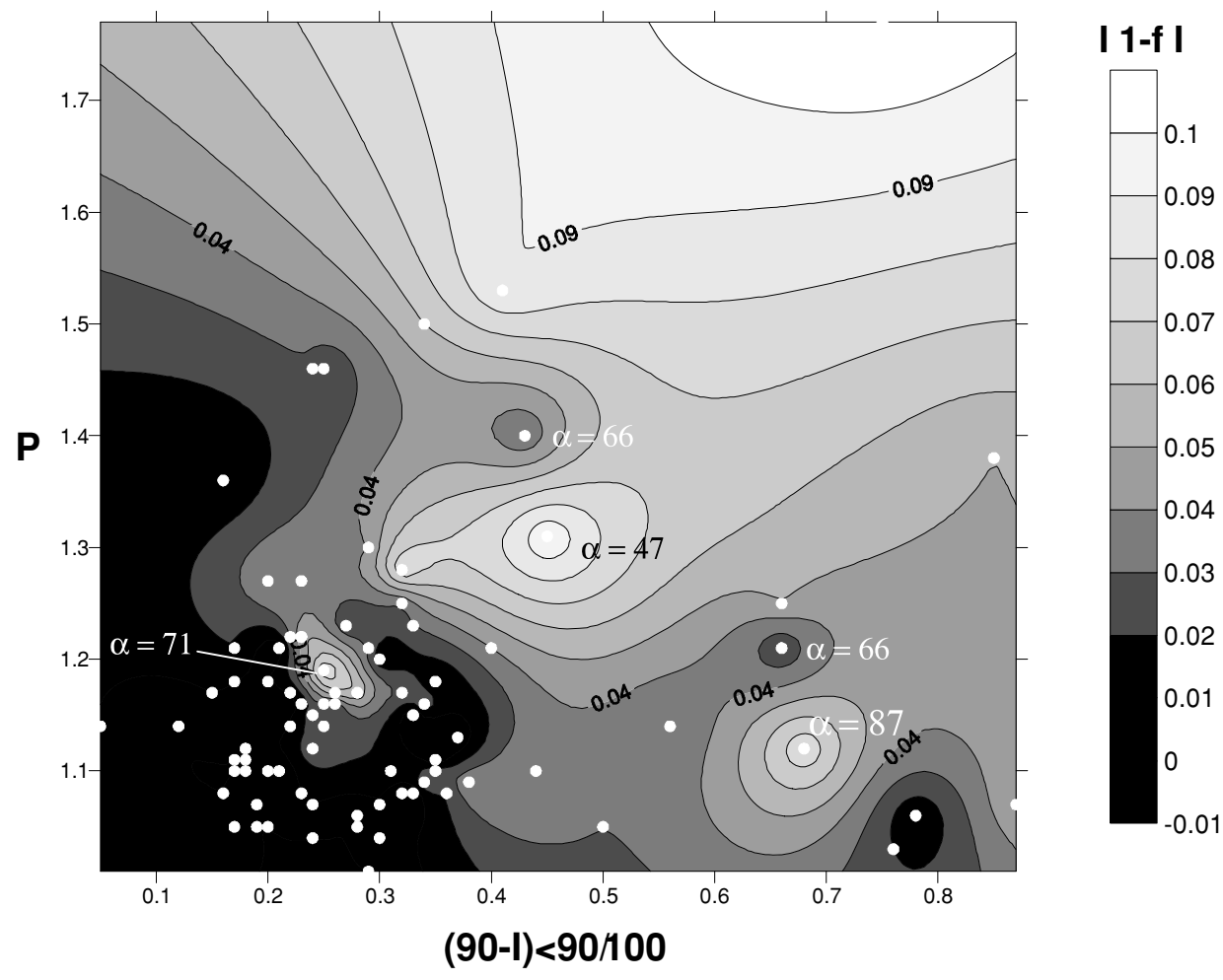

Fig. 11. Contour-plot representing the mutual influence of the degree of TRM anisotropy $P$ and the bias of laboratory field from the direction of carried NRM: $\left(90-I_{\mathrm{NRM}}\right)$ on the anisotropy correction given as $|1-f|$. As in Fig. 10, the values of $\left(90-I_{\mathrm{NRM}}\right)$ have been divided by 100 for better visualization.

tions, as done here, is probably not sufficiently precise for the determination of principal axes' directions, especially for samples with a weak anisotropy.

3. A small effect of the magnetic anisotropy on palaeointensity determinations for baked clay/burnt soils materials (BC-BS) is observed using either IRM or TRM tensors. The more time-consuming TRM tensor determination can be replaced by the IRM tensor of anisotropy for such material, which avoids possible mineralogical changes during heating.

4. The shape of the IRM anisotropy ellipsoid is not identical to that of the TRM anisotropy ellipsoid when bricks/tiles (BR-TL) are concerned and the IRM anisotropy cannot replace the TRM anisotropy evaluation for them.

5. The anisotropy correction of the palaeointensity results for the material different from pottery seems negligible (in the all studied samples but six, it is up to $6 \%$ ); however, it improves the inter-samples dispersion for most of the sites studied here, as previously shown for similar materials (Chauvin et al., 2000) and for palaeomagnetic samples with a strong petrofabric (Selkin $e t$ al., 2000).

6. A detailed analysis of the influence of different parameters on the magnetic anisotropy correction on the palaeointensity values has shown that for the material used in this study, the correction is more sensitive to the degree of TRM anisotropy and to the angular distance between the laboratory field and NRM directions than to the angle between the NRM and the axis of maximum remanence anisotropy ellipsoid.
Acknowledgments. One of the authors (M.K.) expresses her gratitude to the Geosciences Laboratory (University of Rennes 1), where she has been granted leave to carry out 2 months of experimental work. The authors acknowledge their thanks to Dr. Lucien Daly from the IPGP laboratory in Saint-Maur for the useful discussions over the obtained results on the anisotropy. Special thanks are due to Dr. B. Henry for his comments on the initial version of the text and for his efforts and those of one anonymous reviewer on the thorough revision and valuable suggestions for the present manuscript. This helped considerably for further amelioration of the text and illustrations. Thanks are also due to the EPS journal's editor Dr. Masayuki Hyodo for his support and valuable advice during the long process of revisions and corrections. The experimental work was begun 1997 and has continued through the bilateral collaboration between DRI/CNRS-France and Bulgarian Academy of Sciences. The study has been partially supported by the AARCH EU contract No HPRN-CT-2002-00219 (Archaeomagnetic applications for the rescue of cultural heritage).

\section{References}

Aitken, M. J., P. A. Alcock, G. D. Bussel, and C. J. Shaw, Archaeomagnetic determination of the past geomagnetic intensity using ancient ceramics: allowance for anisotropy, Archaeometry, 23(1), 53-64, 1981.

Bowles, J., J. Gee, J. Hildebrand, and L. Tauxe, Archaeomagnetic intensity results from California and Ecuador: evaluation of regional data, Earth Planet. Sci. Lett., 203, 967-981, 2002.

Carvallo, C. and D. J. Dunlop, Archeomagnetism of potsherds from Grand Banks, Ontario: a test of low paleointensities in Ontario around A.D. 1000, Earth Planet. Sci. Lett., 186, 437-450, 2001.

Coe, R. S., S. Gromme, and E. A. Mankinen, Geomagnetic paleointensities from radiocarbon-dated lava flows on Hawaii and the question of the Pacific nondipole low, J. Geophys. Res., 83, 1740-1756, 1978.

Chauvin, A., P. Y. Gillot, and N. Bonhommet, Palaeointensity of the Earth's magnetic Field, recorded by two Late Quaternary sequences at the Island of La Reunion (Indian Ocean), J. Geophys. Res., 96(B2), 1981-2006, 1991.

Chauvin, A., Y. Garcia, Ph. Lanos, and F. Laubenhaimer, Paleointensity of 
the geomagnetic field recovered on archaeomagnetic sites from France, Phys. Earth Planet. Inter., 120, 111-136, 2000.

Daly, L. and H. Zinsser, Etude comparative des anisotropies de susceptibilite et d'aimentation remanente isotherme. Consequences pour l'analyse structurale et le paleomagnetisme, Ann. Geophys., 29, 189200, 1973.

Garcia, Y., Variation de l'intensite du champ magnetique en France durant les deux derniers millenaires, PhD Thesis, University of Rennes 1, 1996.

Genevey, A. and Y. Gallet, Intensity of the geomagnetic field in Western Europe over the past 2000 years: New data from ancient French pottery, J. Geophys. Res., 107(B11), 2285, doi: 10.1029/2001 JB000701, 2002.

Genevey, A., Y. Gallet, and J.-Cl. Margeueron, Eigth thousand years of geomagnetic field intensity variations in the eastern Mediterranean, $J$. Geophys. Res., 108(B5), 2228, doi: 10.1029/2001 JB001612, 2003.

Gomez-Paccard, M., A. Chauvin, Ph. Lanos, J. Thiriot, and P. Jimenez-Castillo, Archeomagnetic study of seven contemporaneous kilns from Murcia (Spain), Phys. Earth Planet. Inter., 157, 16-32, doi:10.1016/j.pepi.2006.03.001, 2006.

Goulpeau, L., Ph. Lanos, and L. Langouet, The anisotropy as a disturbance of the archaeomagnetic dating method, in Proc. 25th Int. Symposium of Archaeometry, 54-58, Elsevier, 1989.

Gram-Jensen, M., N. Abrahamsen, and A. Chauvin, Archaeomagnetic Intensity in Denmark, Phys. Chem. Earth (A), 25(5), 525-531, 2000.

Henry, B., D. Jordanova, N. Jordanova, Ch. Souque, and Ph. Robion, Anisotropy of magnetic susceptibility of heated rocks, Tectonophysics, 366, 241-258, 2003.

Hrouda, F., Magnetic anisotropy of rocks and its application in geology and geophysics, Geophys. Surv., 5, 37-82, 1982.

Hus, J., Anisotropy of TRM, in Abstracts book of the 2nd meeting of AARCH (Archaeomagnetism Applied for Rescue Cultural Heritages), Leoben, September, 2001.

Hus, J., S. Ech-Chakrouni, and D. Jordanova, Origin of magnetic fabric in bricks: its implications in archaeomagnetism, Phys. Chem. Earth, 27(25-31), 1319-1331, 2002.

Jelinek, V., Characterisation of the magnetic fabric of rocks, Tectonophysics, 79, 63-67, 1981.

Jordanova, N., Rock magnetic studies in archaeomagnetism and their contribution to the problem of reliable determination of the ancient geomagnetic field intensity, PhD Thesis, Sofia, 1996 (in Bulgarian).

Jordanova, N., V. Karloukovski, and V. Spataras, Magnetic anisotropy studies on Greek pottery and bricks, Bulg. Geophys. J., 21(4), 49-58, 1995.

Jordanova, N., E. Petrovsky, and M. Kovacheva, Preliminary rock magnetic study of archaeomagnetic samples from Bulgarian prehistoric sites, J. Geomag. Geoelectr., 49, 543-566, 1997.

Jordanova, N., M. Kovacheva, I. Hedley, and M. Kostadinova, On the suitability of baked clay for archaeomagnetic studies as deduced from detailed rock-magnetic studies, Geophys. J. Int., 153, 146-158, 2003.

Kovacheva, M., N. Jordanova, and V. Karloukovski, Geomagnetic field variations as determined from Bulgarian Archaeomagnetic Data. Part II: The Last 8000 Years, Surv. Geophys., 19, 431-460, 1998.

Lanos, $\mathrm{Ph}$., The effect of demagnetising field on thermoremanent magnetization acquired by parallel-sided baked clay blocks, Geophys. J. R. Astron. Soc., 91, 985-1012, 1987a.
Lanos, Ph., Archéomagnétisme des materiaux déplacés, applications à la datation des des matériaux de construction d'argile cuite en archéologie, Thèse de Doctorat, Université de Rennes 1, Rennes, 1987b.

Le Goff, M. and Y. Gallet, A new three-axis vibrating sample magnetometer for continuous high-temperature magnetization measurements: applications to paleo- and archeo-intensity determinations, Earth Planet. Sci. Lett., 229, 31-43, 2004.

Leino, M. A. H. and L. J. Pesonen, Archaeomagnetic intensity in Finland-the last 6400 years, Open File Report Q19/22.0/1994/1, 1994.

Marton, P., Archaeomagnetic directions: the Hungarian calibration curve, in Palaeomagnetism and Tectonics of the Mediterranean Region, edited by A. Morris and D. Tarling, 385-399, Geological Soc., Special Publication No 105, 1996.

Nagata, T., Y. Arai, and K. Momose, Secular variation of the Geomagnetic Total Force during the Last 5000 Years, J. Geophys. Res., 68(18), 5277$5281,1963$.

Odah, H., A. G. Hussain, V. Hoffmann, H. C. Soffel, M. El-Gamili, and H. Deebes, Effect of magnetic anisotropy on the experimentally determined palaeointensity of the geomagnetic field, Earth Planets Space, 53, 363-371, 2001.

Rogers, J., J. M. W. Fox, and M. J. Aitken, Magnetic anisotropy in ancient pottery, Nature, 277(5698), 644-646, 1979.

Selkin, P. A., J. S. Gee, L. Tauxe, W. P. Meurer, and A. J. Newell, The effect of remanence anisotropy on paleointensity estimates: a case from Archean Stillwater Complex, Earth Planet. Sci. Lett., 183, 403-416, 2000 .

Stephenson, A., S. Sadikun, and D. K. Porter, A theoretical and experimental comparison of the anisotropies of magnetic susceptibility and remanence in rocks and minerals, Geophys. J. R. Astron. Soc., 84, 185200, 1986.

Tarling, D. H. and F. Hrouda, The magnetic anisotropy of Rocks, Chapman and Hall, 1993.

Thellier, E. and O. Thellier, Sur l'intensite du shamp magnetique terrestre dans le passe historique et geologique, Ann. Géophys., 15, 285-376, 1959.

Veitch, R. J., I. G. Hedley, and J. J. Wagner, An investigation of the intensity of the geomagnetic field during roman times using magnetically anisotropic bricks and tiles, Arch. Sci. Geneve, 37, Fasc. 3, 359-373, 1984.

Yang, S., J. Shaw, and T. Rolph, Archaeomagnetic studies of Peruvian pottery-from $1200 \mathrm{BC}$ to $1800 \mathrm{AD}$, J. Geomag. Geoelectr., 45, 11931207, 1993a.

Yang, S., J. Shaw, and Q. Wei, A comparison of archaeointensity results from Chinese ceramics using Thellier's and Shaw's palaeointensity methods, Geophys. J. Int., 113, 499-508, 1993b.

Yu, Y. and D. Dunlop, Archaeomagnetism of Ontario potsherds from the last 2000 years, J. Geophys. Res., 105(B8), 19419-19433, 2000.

Yu, Y., D. Dunlop, L. Pavlish, and M. Cooper, Paleointensity determination on the Late Precambrian Tudor Gabbro, Ontario, J. Geophys. Res., 106(B11), 26331-26343, 2001.

M. Kovacheva (e-mail: marykov@abv.bg), A. Chauvin, N. Jordanova, P. Lanos, and V. Karloukovski 